\title{
FD-ZKF : A Zonotopic Kalman Filter Optimizing Fault Detection rather than State Estimation
}

\author{
Masoud Pourasghar ${ }^{a, *}$, Christophe Combastel $^{b}$, Vicenç Puig $^{a}$, Carlos Ocampo-Martinez $^{a}$ \\ ${ }^{a}$ Universitat Politècnica de Catalunya, Institut de Robòtica i Informàtica Industrial (CSIC-UPC), Supervision, Safety and Automatic Control \\ Research Center (CS2AC), C/. Llorens i Artigas 4-6, 08028 Barcelona, Spain. \\ ${ }^{b}$ University of Bordeaux, CNRS, IMS, UMR 5218, 33405 Talence, France
}

\begin{abstract}
Enhancing the sensitivity to faults with respect to disturbances, rather than optimizing the precision of the state estimation using Kalman Filters (KF) is the subject of this paper. The stochastic paradigm (KF) is based on minimizing the trace of the state estimation error covariance. In the context of the bounded-error paradigm using Zonotopic Kalman Filters (ZKF), this is analog to minimize the covariation trace. From this analogy and keeping a similar observerbased structure as in ZKF, a criterion jointly inspired by set-membership approaches and approximate decoupling techniques coming from parity-space residual generation is proposed. Its on-line maximization provides an optimal time-varying observer gain leading to the so-called FD-ZKF filter that allows enhancing the fault detection properties. The characterization of minimum detectable fault magnitude is done based on a sensitivity analysis. The effect of the uncertainty is addressed using a set-membership approach and a zonotopic representation reducing set operations to simple matrix calculations. A case study based on a quadruple-tank system is used both to illustrate and compare the effectiveness of the results obtained from the FD-ZKF approach compared to a pure ZKF approach.
\end{abstract}

Keywords: Uncertain systems, observers, fault detection, bounded uncertainties, zonotopes, sensitivity analysis, minimum detectable fault.

\section{Introduction}

Fault Detection (FD) is a key feature to increase safety and reliability of complex automatic control systems [1]. Model-based FD relies on the use of a model describing the system behavior in order to check the consistency with the observations obtained from some sensors. Therefore, the performance of FD depends on the quality of the mathematical model [1, 2]. The mismatch between the modeled and real behaviors of the system is often nonnegligible when modeling dynamic systems [3]. Relevant description of the uncertainties has been thought of as a key factor in modeling the system dynamic. Consequently, there is a large volume of published studies dealing with the design techniques to explicitly represent such uncertainties in the models [4, 5, 6, 7] and also exploring the influence of the uncertainties in control area [8, 9]. There are two main paradigms in order to represent the uncertainties: the stochastic approaches consider that the uncertainties can be represented using a random variable [10, 11] and the so-called deterministic approaches consider that the uncertainties are unknown but bounded [12, 13].

Generally speaking, model-based FD can be categorized considering how residuals are generated: observers (as e.g. Kalman filter or unknown input observers), parity equations and parameter estimation [2, 14]. Mainly, the state estimation in observer-based approaches is based on the measurements using either stochastic (e.g., Kalman filters) or deterministic approaches (e.g., Luenberger observers) to characterize the propagation of uncertainties. Then, the decision of the FD module can be made based on evaluating the residual generated by using the output estimation error [2, 15]. In particular, in the Kalman filter-based approach, the innovation is considered as a residual to detect the

${ }^{*}$ Corresponding author: Masoud Pourasghar (mpourasgharlaf@iri.upc.edu). 
fault (i.e., in the presence of faults, the prediction error is not null). In this framework, Kalman filter, extended Kalman filter and unscented Kalman filter are different techniques for state estimation of linear/nonlinear systems where the uncertainties are taken into account stochastically [10, 16]. In the set-membership approach, the uncertainties are assumed unknown but bounded [4, 17, 18, 19]. In the case of the set-membership-based state estimation [5, 20, 21], the estimation characterizes a set of possible states. In literature, several families of geometrical structures have been used as e.g. interval boxes, polyhedrons/polytopes, ellipsoids and zonotopes [5, 22, 23, 24].

As in the observer-based approaches, FD using parity equations is based on checking possible inconsistencies of the measured outputs with suitable analytical redundancy relations derived from the system model [25, 26, 27]. On the other hand, it is possible to detect the fault based on the parameter estimation where the plant is firstly identified in fault-free scenario (called reference model). Then, the existence of the fault is proved by checking the reference model with the parameters that are repeatedly re-identified on-line [28, 29, 30].

Some works have explored the relation between different approaches regarding FD performance [14, 31, 32]: for example, if the residual generators have been designed for the same specification, parity relation and observer-based approaches can produce identical residuals.

A large and growing amount of literature has also reported several approaches to improve the FD methods such that the residual is obtained to be insensitive to the uncertainty, while at the same time sensitive to the faults [2, 27, 33]. This is the case of FD filters that are designed by considering the robustness against disturbance, noise or any uncertainties using, e.g., $H_{\infty}$ optimization, Linear Matrix Inequalities (LMI)s or $\mu$ design techniques [33, 34]. But, recent results show that the filter design technique considering only the rejection of the effect of uncertainties has not been successful since the sensitivity to the fault needs to be considered in FD filter design [35]. This is the case of [36] where the multi-objective FD design based on $H_{-} / H_{\infty}$ techniques is considered. Indeed, the worst case of the fault sensitivity is taken into account by the smallest not null singular value of the transfer function matrix from fault to residual at either $\omega=0$ or over a given frequency range [37, 38]. Then, a significant amount of literature has been published discussing this multi-objective design task formulated as an optimization problem, e.g., $H_{\infty} / H_{\infty}, H_{2} / H_{\infty}$ or $\mathrm{H}_{2} / \mathrm{H}_{2}$ problems [35, 39, 40, 41].

On the other hand, a considerable amount of literature has been reported on different state-bounding algorithms based on stochastic and deterministic approaches. A recent study, proposed by [42], deals with the standard Kalman filtering together with the zonotopic state estimation. This work establishes an explicit bridge between stochastic/Gaussian and set-based/zonotopic frameworks relying on the analogy between covariances and covariation. Then, in [43] and [44], both deterministic (bounded) and stochastic (Gaussian) disturbances have been taken into account in order to propose the extended version of Zonotopic and Gaussian Kalman Filter (ZGKF) with the aim of merging Gaussian Kalman filtering and zonotopic state bounding to achieve robust FD. Furthermore, there is a large volume of reported studies about fault detectability, in particular [45], where the main objective is to compute the minimum magnitude of the fault that can be detected when an interval observer is used.

The main contribution of this paper is to make use of a similar observer-based structure as in ZKF in order to propose an approach enhancing the sensitivity to faults with respect to disturbances, rather than only optimizing the precision of the state estimation as is usually considered in Kalman Filters. As for ZKF, a generic discrete-time linear time-varying (LTV) dynamic model of the system is considered. Contrary to most existing multi-objective FD design techniques, the sensitivity to both faults and disturbances is evaluated using a set-based approach enclosing all the possible temporal scenarios of faults and disturbances within specified ranges. Moreover, the proposed criterion combining the sensitivity to both faults and disturbances makes it possible to efficiently obtain on-line time-varying optimal FD observer gains, without any requirement on the considered frequency ranges. The combination of these features makes the approach original compared to most multi-objective optimization-based FD techniques. Finally, the effectiveness of the proposed approach is illustrated using a case study based on quadruple-tank system.

Regarding the structure of the paper, some preliminaries are introduced in Section 2. The problem formulation is addressed in Section 3 The observer structure and the FD algorithm are discussed in Section 4 The computation of a time-varying observer gain optimizing a set-based criterion modeling the trade-off between the sensitivity to faults and the robustness to disturbances is proposed in Section 5. The discussion of the comparative assessment and the characterization of the minimum detectable fault for the FD-ZKF and ZKF approaches are presented in Section 6. The application to a quadruple-tank system is used in order to illustrate the effectiveness of the proposed approach in Section 7. Finally, conclusions are drawn in Section 8 . 


\section{Preliminaries}

\subsection{Notation}

Throughout this paper, $\mathbb{R}^{n}$ denotes the set of $n$-dimensional real numbers, $\oplus$ denotes the Minkowski sum, $\otimes$ indicates the Kronecker product. The matrices are written using capital letter, e.g., $A$, the calligraphic notation is used for denoting sets, e.g., $\mathcal{X}$, the transfer functions are highlighted using script font e.g., $\mathscr{H},\|.\|_{s}$ denotes the $s$-norm and $[\underline{x}, \bar{x}]$ is an interval with lower bound $\underline{x}$ and upper bound $\bar{x}$.

\subsection{Zonotope and set operation}

Definition 1. (Zonotope) A zonotope $\langle c, R\rangle \subset \mathbb{R}^{n}$ with the center $c \in \mathbb{R}^{n}$ and the generator matrix $R \in \mathbb{R}^{n \times p}$ is a polytopic set defined as a linear image of the unit hypercube $[-1,1]^{p}$ :

$$
\langle c, R\rangle=\left\{c+R s,\|s\|_{\infty} \leq 1\right\} .
$$

Moreover, a centered zonotope is denoted by $\langle R\rangle=\langle 0, R\rangle$. Any permutation of the columns of $R$ leaves it invariant. For example, Figure 1 illustrates the sixth-order zonotope in $\mathbb{R}^{3}$ with $c=\left[\begin{array}{l}0 \\ 0 \\ 0\end{array}\right]$ and $R=\left[\begin{array}{cccccc}1 & 1 & 1 & -1 & 0 & 0 \\ 1 & -1 & 0 & 0 & 1 & -1 \\ 0 & 0 & -1 & -1 & 1 & 1\end{array}\right]$.

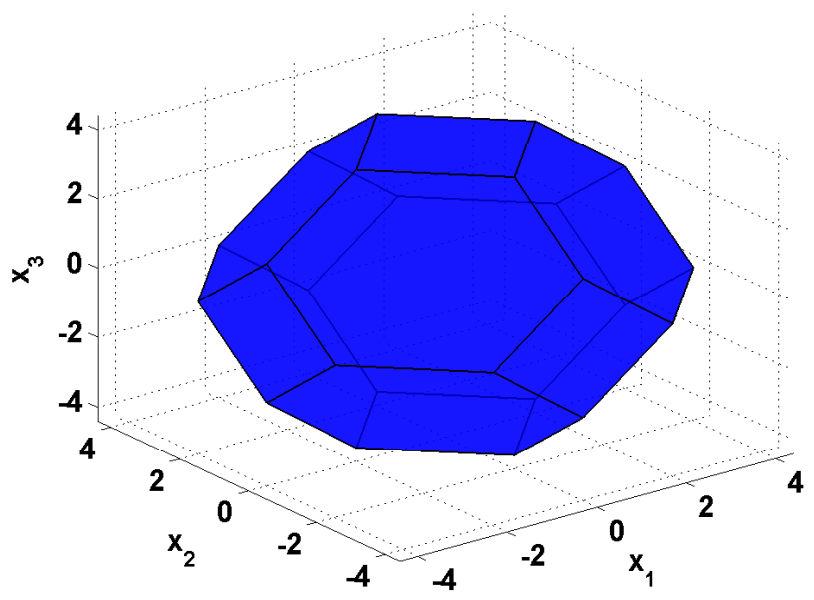

Figure 1: Zonotope in $\mathbb{R}^{3}$

Definition 2. (Minkowski sum) Considering two sets $\mathcal{A}$ and $\mathcal{B}$, their Minkowski sum is a set defined as $\mathcal{A} \oplus \mathcal{B}=$ $\{a+b \mid a \in \mathcal{A}, b \in \mathcal{B}\}$. Thus, the Minkowski sum of the zonotopes $\mathcal{Z}_{1}=\left\langle c_{1}, R_{1}\right\rangle$ and $\mathcal{Z}_{2}=\left\langle c_{2}, R_{2}\right\rangle$ is $\mathcal{Z}_{1} \oplus \mathcal{Z}_{2}=$ $\left\langle c_{1}+c_{2},\left[R_{1}, \quad R_{2}\right]\right\rangle$.

Definition 3. (F-radius) The $F$-radius of a zonotope $\mathcal{Z}=\langle c, R\rangle$ is the Frobenius norm of its shape matrix, i.e., $\|R\|_{F}$.

Definition 4. (Covariation) The covariation of the zonotope $\mathcal{Z}=\langle c, R\rangle$ is $\operatorname{cov}(\langle c, R\rangle)=R R^{T}$ [42, 43].

Property 1. (Linear image) The linear image of a zonotope $\mathcal{Z}=\langle c, R\rangle$ by a matrix $L$ with a compatible size is $L \odot\langle c, R\rangle=\langle L c, L R\rangle$.

Property 2. (Reduction operator) A reduction operator denoted $\downarrow_{q}$ permits to reduce the number of generators of a zonotope $\langle c, R\rangle$ to a fixed number $q$ while preserving the inclusion property $\langle c, R\rangle \subset\left\langle c, \downarrow_{q} R\right\rangle$. A simple yet efficient solution to compute $\downarrow_{q} R$ is given in [24, 42]. It consists in sorting the columns of $R$ on decreasing Euclidean norm and enclosing the influence of the smaller columns only into an easily computable interval hull, so that the resulting matrix $\downarrow_{q} R$ has no more than $q$ columns. 


\section{Problem Formulation}

This paper addresses the problem of FD in dynamical systems modeled as an uncertain time-varying state-space representation in discrete-time as

$$
\begin{array}{r}
x_{k+1}=A_{k} x_{k}+B_{u, k} u_{k}+E_{k} v_{k}, \\
y_{k}=C_{k} x_{k}+D_{u, k} u_{k}+F_{k} v_{k},
\end{array}
$$

where $k \in \mathbb{N}$ indicates the discrete time. Furthermore, $x \in \mathbb{R}^{n_{x}}$ is the state vector, $u \in \mathbb{R}^{n_{u}}$ and $y \in \mathbb{R}^{n_{y}}$ denote the known input and the known output (measurement) vectors, respectively. The system matrices of appropriate dimensions are $A \in \mathbb{R}^{n_{x} \times n_{x}}, B_{u} \in \mathbb{R}^{n_{x} \times n_{u}}, C \in \mathbb{R}^{n_{y} \times n_{x}}$ and $D_{u} \in \mathbb{R}^{n_{x} \times n_{u}}$. Moreover, the random vector $v \in \mathbb{R}^{n_{v}}$ denotes an additive uncertainty that is bounded by a unit hypercube expressed as a centered zonotope, i.e., $\forall k \geq 0, v_{k} \in$ $\left[\begin{array}{ll}-1, & 1\end{array}\right]^{n_{v}}=\left\langle 0, I_{n_{v}}\right\rangle$, where $I_{n_{v}} \in \mathbb{R}^{n_{v} \times n_{v}}$ denotes the identity matrix. Furthermore, $E$ and $F$ are non-empty matrices with appropriate dimensions.

Moreover, the bounded uncertainty vector $v_{k}$ is considered as the combination of the disturbance and the fault. Therefore, the decomposed form of $v_{k}$ is written as

$$
v_{k}=\left[\begin{array}{l}
d_{k} \\
f_{k}
\end{array}\right],
$$

where $d \in[-1,1]^{n_{d}}=\left\langle 0, I_{n_{d}}\right\rangle$ and $f \in[-1,1]^{n_{f}}=\left\langle 0, I_{n_{f}}\right\rangle$, respectively, modeling the disturbances and faults that possibly influence the system. Consequently, $E_{k}$ and $F_{k}$ can be decomposed as

$$
\begin{aligned}
E_{k} & =\left[\begin{array}{ll}
B_{d, k} & B_{f, k}
\end{array}\right], \\
F_{k} & =\left[\begin{array}{ll}
D_{d, k} & D_{f, k}
\end{array}\right],
\end{aligned}
$$

where $B_{d}, B_{f}, D_{d}$ and $D_{f}$ denote non-empty matrices with suitable dimensions.

Henceforth, the index $k+1$ will be replaced by + and $k$ will be omitted for the sake of simplified notations. Then, the dynamical model (2) is simply rewritten while remaining fully LTV 1 as

$$
\begin{gathered}
x_{+}=A x+B_{u} u+E v, \\
y=C x+D_{u} u+F v .
\end{gathered}
$$

The initial state $x_{0}$ belongs to the zonotopic set $\mathcal{X}_{0}=\left\langle c_{0}, R_{0}\right\rangle$, where $c_{0} \in \mathbb{R}^{n_{x}}$ denotes the center and $R_{0} \in \mathbb{R}^{n_{x} \times r_{R_{0}}}$ is a non-empty matrix containing the generators matrix $R_{0}$ of the initial zonotope $\mathcal{X}_{0}$. The pair $(A, C)$ is assumed to be detectable. Moreover, monitoring the system with the dynamical model (3) considering the influence of disturbances and the possible effect of the fault (when it exists) can be done deriving a set-membership observer from the expression

$$
x_{+}=A x+B_{u} u+E v+G\left(y-C x-D_{u} u-F v\right),
$$

where $G$ is the observer gain that provides degrees of freedom to tune the system monitoring with respect to its aim, i.e., with the goal of optimizing the detection of faults according to some given criterion.

In order to highlight the link with an observer that will be formalized in Section 4.1 it can be noticed that replacing the variables in the right-hand side of (4) by the zonotope they belong to, the terms corresponding to centers will result in a classical Luenberger observer with observer gain $G[5$ a $]$, while the generator/shape matrix terms will provide an explicit way to parameterize with $G$ the zonotopic enclosures of the classical observation error $(5 \mathrm{~b})$.

An optimal tuning of the time-varying observer gain matrix $G$ based on a set-based optimization criterion expressing the desired FD performance (rather than the only state estimation) is addressed in the following of the paper.

${ }^{1}$ LTV : Linear Time-Varying 


\section{Structure of the observer-based FD tests}

\subsection{General observer structure}

In this paper, the observer-based FD is performed by means of a Kalman filter using zonotopic sets instead of Gaussian probability density functions (PDF), known as Zonotopic Kalman Filter (ZKF). Considering the one-step ahead predictor form, also called delayed form of the Kalman filter, the underlying observer structure is determined using the ZKF approach proposed in [42], which can be further tuned with respect to the FD purpose to increase the FD performance. Therefore, the following Proposition 4.1 is obtained based on (4).

Proposition 4.1. (Observer structure) Considering the dynamical model (3), the center $c$ and the shape matrix $R$ of the zonotopic observer can be recursively defined as

$$
\begin{aligned}
& c_{+}=(A-G C) c+\left(B_{u}-G D_{u}\right) u+G y,
\end{aligned}
$$

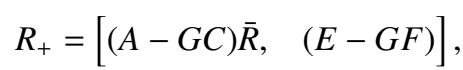

where $\bar{R}=\downarrow_{q} R$. Furthermore, the state inclusion property $x \in\langle c, R\rangle$ holds for all $k \geq 0$.

Proof. Assuming $x \in\langle c, R\rangle$ and $v \in\left\langle 0, I_{n_{v}}\right\rangle$ for all $k \geq 0$ where the inclusion property is preserved, (4) can be written using the reduction operator as

$$
x_{+} \in\left\langle c_{+}, R_{+}\right\rangle=\langle(A-G C) c,(A-G C) \bar{R}\rangle \oplus\left\langle\left(B_{u}-G D_{u}\right) u, 0\right\rangle \oplus\langle 0,(E-G F)\rangle \oplus\langle G y, 0\rangle .
$$

Thus, based on Definition 2 and Property 1, the center $c_{+}$and the shape matrix $R_{+}$in (6) can be expressed as in (5), where the center $c$ can be interpreted as a classical punctual state estimate of the unknown state $x$ and the shape matrix $R$ characterizes a zonotopic enclosure of the classical observation error $e=x-c$.

\subsection{FD based on the innovation term}

The standard form of FD test is based on checking the consistency of the measurements with a fault-free model. In this work, the fault-free model is obtained by setting $f=0$ in (2). Then, $v=\left[\begin{array}{l}d \\ 0\end{array}\right]$.

The innovation is usually defined as the difference between the measured value of a variable at time $k$ and the optimal forecast of that value based on the information available prior to time $k$. In this work, the measured value of the output is $y$, and $c$, which will result from iterations based on (5a) with some optimal $G$, stands for the above mentioned optimal forecast in the considered one-step ahead predictor form (or delayed form) of KF. The reader interested in additional material about formal/detailed links between the set-membership and stochastic paradigms and their joint use for the design of some innovation-based FD tests is referred to [42] and [43, 44], respectively. In particular, explicit links with the computation and evaluation forms of prediction error/residuals are formalized in [43, 44].

Proposition 4.2. (FD test design) Considering a faultless scenario $(f=0)$, the center $c_{\varepsilon}$ and the shape matrix $R_{\varepsilon}$ of a zonotope containing the origin 0 , i.e., satisfying $0 \in\left\langle c_{\epsilon}, R_{\epsilon}\right\rangle$ at time $k$ is

$$
\begin{aligned}
& c_{\varepsilon}=y-\left(C c+D_{u} u\right), \\
& R_{\varepsilon}=\left[\begin{array}{ll}
-C R, & -F
\end{array}\right] .
\end{aligned}
$$

Proof. The output equation $3 \mathrm{~b}$ can be rewritten as follows:

$$
0=y-C x-D_{u} u-F v
$$

Substituting $x \in\langle c, R\rangle$ for $x$ in (8) and $v \in\left\langle 0, I_{n_{v}}\right\rangle$ yields

$$
0 \in\langle y, 0\rangle \oplus\langle-C c,-C R\rangle \oplus\left\langle-D_{u} u, 0\right\rangle \oplus\langle 0,-F\rangle,
$$


which completes the proof by applying Definition 2 and Property 1.

It is worth noting that the time-varying center $c_{\varepsilon}$ can be equivalently identified/interpreted at time $k$ as:

i) a prediction error which is homogeneous to the system output in terms of physical units,

ii) a residual $r$ whose computation form corresponds to the right term of $(7 \mathrm{a})$, and

iii) an innovation term $\varepsilon$, as explained in the text introducing the Proposition 4.2

Since $\left\langle 0, R_{\varepsilon}\right\rangle=\left\langle R_{\varepsilon}\right\rangle$ is a centered zonotope and, as such, a centrally symmetric domain, and since $\varepsilon=r=c_{\varepsilon}$, it can be noticed that $0 \in\left\langle c_{\varepsilon}, R_{\varepsilon}\right\rangle$ as stated in Proposition 4.2 equivalently can be rewritten as $\varepsilon \in\left\langle R_{\varepsilon}\right\rangle$ and/or $r \in\left\langle R_{\varepsilon}\right\rangle$. As a result, the zonotope shape matrix $R_{\varepsilon}(7 \mathrm{~b})$ gives explicit information for the evaluation of the residual $r$.

Therefore, the FD test is done by computing (7) and the fault is detected when $0 \notin\left\langle c_{\varepsilon}, R_{\varepsilon}\right\rangle$. A computationally efficient way to implement the detection test without increasing the false alarm rate consists in testing whether 0 belongs or not to an aligned box enclosing the zonotope $\left\langle c_{\varepsilon}, R_{\varepsilon}\right\rangle$

$$
0 \notin\left\langle c_{\varepsilon}, b\left(R_{\varepsilon}\right)\right\rangle,
$$

where $\left\langle c_{\varepsilon}, b\left(R_{\varepsilon}\right)\right\rangle$ is enclosed by an align box characterized by $b\left(R_{\varepsilon}\right)=\operatorname{diag}\left(\left|R_{\varepsilon}\right| \mathbf{1}\right)$. Considering that $|$.$| is the element-$ by-element absolute value operator, $\mathbf{1}$ is a column vector of ones and $\operatorname{diag}($.$) returns a diagonal matrix from a vector$ of diagonal elements.

Algorithm 1 summarizes the FD test in Proposition 4.2 .

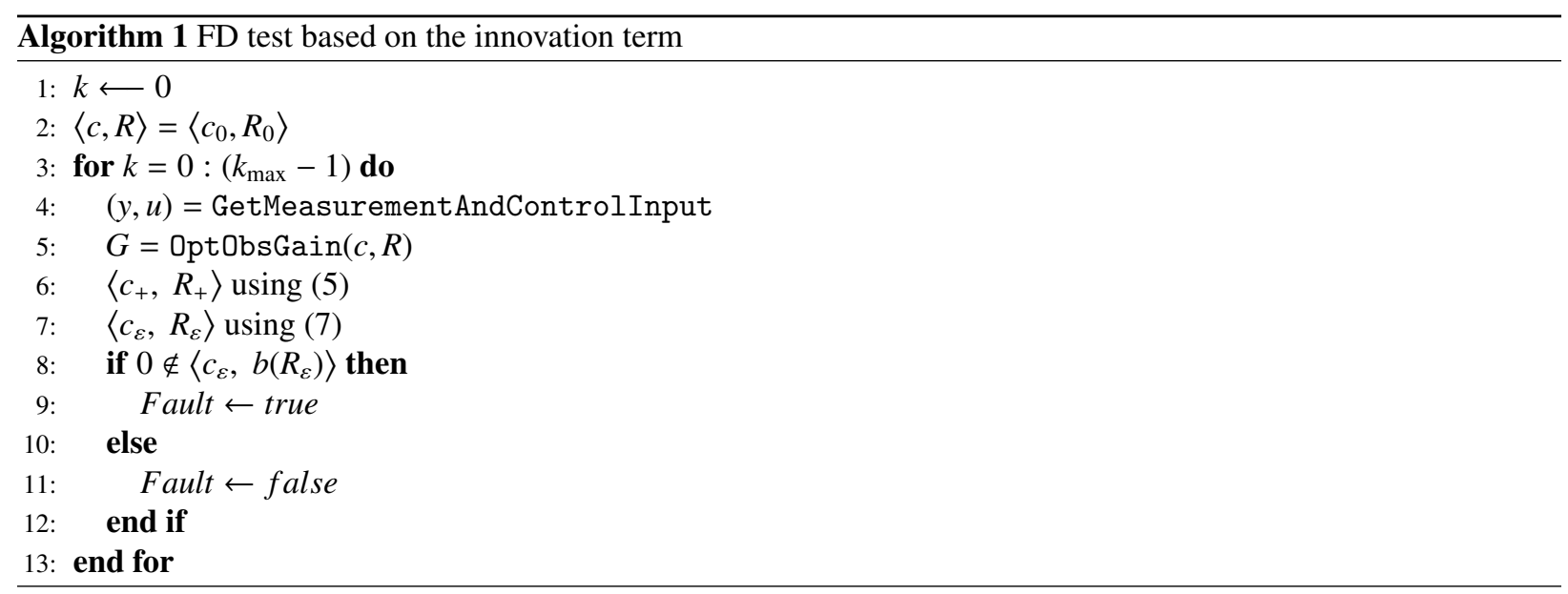

Note that the first step of Algorithm 1 is related to initialization. Then, the explicit value of the optimal observer gain should be computed using either an observation-based or an FD-based criterion. Moreover, a function $G=$ OptObsGain $(c, R)$ implementing such computations will be presented later in the paper. Then, the one step ahead state prediction $\left\langle c_{+}, R_{+}\right\rangle$as well as the value of the residual vector $r=c_{\varepsilon}$ and its adaptive threshold $b\left(R_{\varepsilon}\right)$ can be computed based on $u, y$ and $G$. So, the FD test is based on computing the next-step bounding set with the computed optimal gain and Proposition 4.1. Finally, the residual is generated using Proposition 4.2 and the fault is detected when $0 \notin\left\langle c_{\varepsilon}, b\left(R_{\varepsilon}\right)\right\rangle$.

\section{Optimal zonotopic observer gain}

\subsection{Observer structure}

The observer gain has important implications in the behavior of the state bounding observer resulting from Proposition 4.1. Contrary to a first intuition, it is not clear that those computed observer gains that are suitable for the observation purpose are also suitable for the purpose of FD. In this regard, using the same observer structure, the focus of this section will be placed on first giving a brief overview of computing the observer gain with only state observation purposes. Then, computing the optimal observer gain regarding FD will be considered by using a disturbance/fault reach set-based criterion. 
In order to characterize them as functions of the tuning matrix $G$, the reach sets describing the influence of the disturbances and the effect of a fault on the estimated state sets, the observer structure in Proposition 4.1 can be decomposed and rewritten as shown in Proposition 5.1

Proposition 5.1. (Superposed form of the observer structure) Considering the dynamical model (3), the decomposition of the center $c$ and the shape matrix $R$ of the state bounding observer into the effects of the disturbance and fault can be recursively defined as

$$
x_{+} \in\left\langle c_{d_{+}}, R_{d_{+}}\right\rangle \oplus\left\langle c_{f_{+}}, R_{f_{+}}\right\rangle,
$$

with

$$
\begin{aligned}
c_{d_{+}} & =(A-G C) c_{d}+\left(B_{u}-G D_{u}\right) u+G y, \\
R_{d_{+}} & =\left[(A-G C) \bar{R}_{d}, \quad\left(B_{d}-G D_{d}\right)\right], \\
c_{f_{+}} & =(A-G C) c_{f}, \\
R_{f_{+}} & =\left[(A-G C) \bar{R}_{f}, \quad\left(B_{f}-G D_{f}\right)\right],
\end{aligned}
$$

where $\bar{R}_{d}=\downarrow_{q} R_{d}$ and $\bar{R}_{f}=\downarrow_{q} R_{f}$. Additionally, the inclusion property is preserved for all $k \geq 0$, i.e., $x \in\left\langle c_{d}, R_{d}\right\rangle \oplus$ $\left\langle c_{f}, R_{f}\right\rangle$.

Proof. Assuming $x \in\left\langle c_{d}, R_{d}\right\rangle \oplus\left\langle c_{f}, R_{f}\right\rangle$ at time instant $k, d \in\left\langle 0, I_{n_{d}}\right\rangle$ and $f \in\left\langle 0, I_{n_{f}}\right\rangle$, it is possible to write the zonotopic form of the observer in (4) as

$$
\begin{aligned}
x_{+} \in\left\langle(A-G C) c_{d},(A-G C) R_{d}\right\rangle \oplus\left\langle(A-G C) c_{f},(A-G C) R_{f}\right\rangle \\
\quad \oplus\left\langle\left(B_{u}-G D_{u}\right) u, 0\right\rangle \oplus\langle G y, 0\rangle \oplus\left\langle 0,\left(B_{d}-G D_{d}\right)\right\rangle \oplus\left\langle 0,\left(B_{f}-G D_{f}\right)\right\rangle .
\end{aligned}
$$

Furthermore, consider that the superposition principle can be explicitly invoked in the linear setting. Therefore, using Definition 2, the center and the generator matrix in (13) can be reorganized as in (12). Thus, $x_{+} \in\left\langle c_{d+}, R_{d+}\right\rangle \oplus$ $\left\langle c_{f+}, R_{f+}\right\rangle$. This gives the proof by induction.

Since $x \in\left\langle c_{d}, R_{d}\right\rangle \oplus\left\langle c_{f}, R_{f}\right\rangle$ is independent from the observer gain at time instant $k$, the effect of the disturbance and the fault at time instant $k$ can be reformulated as a one-step prediction from time instant $k-1$ in order to parameterize the effect of the observer gain. Thus, the effect of the disturbance and fault at time instant $k$ in Proposition 5.1 can be formulated as

$$
\begin{aligned}
& c_{d}=\left(A_{-}-G_{-} C_{-}\right) c_{d_{-}}+\left(B_{u_{-}}-G_{-} D_{u_{-}}\right) u_{-}+G_{-} y_{-}, \\
& R_{d}=\left[\begin{array}{ll}
\left(A_{-}-G_{-} C_{-}\right) R_{d_{-}}, \quad\left(B_{d_{-}}-G_{-} D_{d_{-}}\right) & ],
\end{array}\right. \\
& c_{f}=\left(A_{-}-G_{-} C_{-}\right) c_{f_{-}},
\end{aligned}
$$

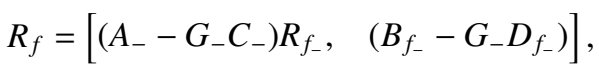

where the subscript - is a short notation of $k-1$. Therefore, the state-bounding zonotope becomes an affine function of the (previous) observer gain $x \in\left\langle c_{d}\left(G_{-}\right), R_{d}\left(G_{-}\right)\right\rangle \oplus\left\langle c_{f}\left(G_{-}\right), R_{f}\left(G_{-}\right)\right\rangle$.

The use of Proposition 5.1 instead of Proposition 4.1 allows the separation of the effects of the disturbances and the faults. Therefore, computing the observer gain for observation purposes is done considering (12a) and (12b) while tuning the observer gain for FD purposes is done considering (12a to (12d). Then, the expected strength of the proposed FD scheme lies in the fact that not only the influence of disturbances is used, but also the relative influence of disturbances and faults can be used to set up an optimization criterion quantifying the satisfaction level of an FD goal.

\subsection{Optimal observer gain for observation purposes}

When designing the gain for state-observation purposes, the main goal will be only reducing the effect of state estimation uncertainty. The computation of the optimal observer gain for an observation purpose is investigated in [42] 
which shows that there is a strong analogy between the Kalman filter and zonotopic Kalman filter (ZKF) where the usually Gaussian probability density functions (PDF) are replaced by zonotopic sets. Therefore, the optimal observer gain $G^{*}$ can be obtained by minimizing the $F$-radius $s^{2}$ of the zonotope $\left\langle c_{+}, R_{+}\right\rangle$in [5]. According to [42], minimizing the $F$-radius of a zonotope is equivalent to minimizing the trace of its covariation. Therefore, considering Definition 4 , an accuracy criterion related to the size of the next state-bounding zonotope can be written as

$$
J=\operatorname{tr}\left(R_{+} R_{+}^{T}\right)=\left\|R_{+}\right\|_{F}^{2} .
$$

Then, considering the fault-free model $(f=0)$ which is the standard approach for a general purpose state observation, $J$ may be expressed as

$$
J=\operatorname{tr}\left(R_{d_{+}} R_{d_{+}}^{T}\right)=\left\|R_{d_{+}}\right\|_{F}^{2} .
$$

Moreover, given the state-bounding zonotope at time instant $k$ as $x \in\langle c, R\rangle, G^{*}$ can be computed explicitly using Theorem 5.1 in order to minimize the effect of uncertainty over the next state-bounding zonotope $x_{+} \in\left\langle c_{+}, R_{+}\right\rangle$.

Theorem 5.1. Considering $x \in\langle c, R\rangle$ at time $k$, the optimal observer gain $G^{*}$ minimizing the $F$-radius of the statebounding zonotope at time instant $k+1$, or precisely, minimizing the criterion $J=\operatorname{tr}\left(R_{+} R_{+}^{T}\right)$ obtained in [16, is computed as

$$
G^{*}=A K^{*}
$$

with

$$
K^{*}=L S^{-1}, \quad L=\bar{P} C^{T}, \quad S=C \bar{P} C^{T}+Q_{\omega},
$$

where the covariation matrices are introduced as $Q_{\omega}=D_{d} D_{d}^{T}$, $Q_{v}=B_{d} B_{d}^{T}$ and $\bar{P}=\bar{R}_{d} \bar{R}_{d}^{T}$.

Proof. The proof follows from the results presented in [42].

\subsection{Optimal observer gain for FD purposes}

Now, this section will concentrate on the design of the observer gain for FD purposes. Such a gain is computed to maximize the effect of faults with respect to disturbances. In this regard, an optimal tuning based on an FD criterion (FD-ZKF) rather than an observation criterion (ZKF) is used in order to enhance the FD properties of the observer. By analogy with the Kalman filter, minimizing the $F$-radius of a zonotope is equivalent to minimizing the trace of its covariation. Therefore, the following accuracy criterion can be written to maximize the influence of faults while minimizing that of disturbances:

$$
J\left(G_{-}\right)=\frac{\operatorname{tr}\left(\operatorname{cov}\left(R_{f}\left(G_{-}\right)\right)\right)}{\operatorname{tr}\left(\operatorname{cov}\left(R_{d}\left(G_{-}\right)\right)\right)} .
$$

Remark 5.1. The criterion proposed in (18) follows a general idea that was inspired by optimal approximate decoupling techniques coming from parity-space residual generation as presented in [27]. Note that the notion of worst-case highly depends on a related evaluation criterion. An intuitive interpretation motivating the use of the original optimization criterion (18) is that it makes it possible to obtain an optimal time-varying observer gain maximizing the size (covariation) of a reachable set describing the influence of all the specified faults while minimizing the size (covariation) of a reachable set describing the influence of all the specified disturbances. This is in contrast with methods maximizing the influence of extreme faults (e.g. those involving the lowest sensitivity in terms of $H_{-}$) while minimizing the influence to extreme disturbances (e.g. those involving the maximal sensitivity expressed in terms of $H_{\infty}$ ). Moreover, the proposed time-varying optimal observer gain can be expressed in a mathematically tractable way and the complexity of its computation remains compatible with online implementations.

Once the optimization criterion based on a matrix parameter is chosen as (18), Proposition 5.2 can be used in order to parameterize the optimization criterion based on a vector $\omega$ rather than a matrix parameter like $G_{-}$.

\footnotetext{
${ }^{2}$ Further information about Frobenius norm can be found in [42].
} 
Proposition 5.2. Considering the definition of matrix trace and introducing $\omega=\operatorname{col}\left(G_{-}\right)$, where col(.) denotes the vertical concatenation of the matrix columns, the FD optimization criterion (18) can be parameterized with a parameter vector as

$$
J\left(G_{-}\right)=\frac{\omega^{T}\left(M_{f}^{T} M_{f}\right) \omega+2 N_{f}^{T} M_{f} \omega+\left(N_{f}^{T} N_{f}\right)}{\omega^{T}\left(M_{d}^{T} M_{d}\right) \omega+2 N_{d}^{T} M_{d} \omega+\left(N_{d}^{T} N_{d}\right)},
$$

with

$$
\begin{aligned}
M_{f}=\left[\begin{array}{c}
-\left(\left(C_{-} R_{f_{-}}\right)^{T} \otimes I\right) \\
-\left(D_{f}^{T}\right) \otimes I
\end{array}\right], & N_{f}=\left[\begin{array}{c}
\operatorname{col}\left(A_{-} R_{f_{-}}\right) \\
\operatorname{col}\left(B_{f_{-}}\right)
\end{array}\right], \\
M_{d}=\left[\begin{array}{c}
-\left(\left(C_{-} R_{d_{-}}\right)^{T} \otimes I\right) \\
-\left(D_{d}^{T}\right) \otimes I
\end{array}\right], & N_{d}=\left[\begin{array}{c}
\operatorname{col}\left(A_{-} R_{d_{-}}\right) \\
\operatorname{col}\left(B_{d_{-}}\right)
\end{array}\right] .
\end{aligned}
$$

Proof. Taking into account the Definition 4, the covariation of the matrices $R_{f}$ and $R_{d}$ can be written as

$$
\begin{aligned}
& \operatorname{cov}\left(R_{f}\left(G_{-}\right)\right)=\left(R_{f}\left(G_{-}\right)\right)\left(R_{f}\left(G_{-}\right)\right)^{T}, \\
& \operatorname{cov}\left(R_{d}\left(G_{-}\right)\right)=\left(R_{d}\left(G_{-}\right)\right)\left(R_{d}\left(G_{-}\right)\right)^{T},
\end{aligned}
$$

where both are quadratic functions of the elements of $G_{-}$. Then, considering the definition of the matrix trace, the trace of both $\operatorname{cov}\left(R_{f}\left(G_{-}\right)\right)$and $\operatorname{cov}\left(R_{d}\left(G_{-}\right)\right)$can be expressed based on column vectors as

$$
\begin{aligned}
& \operatorname{tr}\left(\operatorname{cov}\left(R_{f}\left(G_{-}\right)\right)\right)=\operatorname{col}\left(R_{f}\left(G_{-}\right)\right)^{T} \operatorname{col}\left(R_{f}\left(G_{-}\right)\right), \\
& \operatorname{tr}\left(\operatorname{cov}\left(R_{d}\left(G_{-}\right)\right)\right)=\operatorname{col}\left(R_{d}\left(G_{-}\right)\right)^{T} \operatorname{col}\left(R_{d}\left(G_{-}\right)\right) .
\end{aligned}
$$

Additionally, using Kronecker product to introduce matrices $M$ and $N$ in 20, which are independent matrices with respect to $G_{-}$(for both disturbance and fault cases), the column form of $R_{d}$ and $R_{f}$ can be written as

$$
\begin{aligned}
& \operatorname{col}\left(R_{f}\left(G_{-}\right)\right)=M_{f} \omega+N_{f}, \\
& \operatorname{col}\left(R_{d}\left(G_{-}\right)\right)=M_{d} \omega+N_{d},
\end{aligned}
$$

where the column vector $\omega=\operatorname{col}\left(G_{-}\right)$is obtained by reshaping the observer gain matrix $G_{-}$through a vertical concatenation of its column vectors. Then, the substitution of 23] into 22 yields

$$
\begin{aligned}
\operatorname{tr}\left(\operatorname{cov}\left(R_{f}\left(G_{-}\right)\right)\right) & =\left(\omega^{T} M_{f}^{T}+N_{f}^{T}\right)\left(M_{f} \omega+N_{f}\right) \\
& =\omega^{T}\left(M_{f}^{T} M_{f}\right) \omega+2 N_{f}^{T} M_{f} \omega+\left(N_{f}^{T} N_{f}\right), \\
\operatorname{tr}\left(\operatorname{cov}\left(R_{d}\left(G_{-}\right)\right)\right) & =\left(\omega^{T} M_{d}^{T}+N_{d}^{T}\right)\left(M_{d} \omega+N_{d}\right) \\
& =\omega^{T}\left(M_{d}^{T} M_{d}\right) \omega+2 N_{d}^{T} M_{d} \omega+\left(N_{d}^{T} N_{d}\right) .
\end{aligned}
$$

Therefore, after the substitution of (24) in (18), the parameterized optimization criterion with FD purposes can be obtained as 19. 
Before continuing the analysis, some covariation matrices are first introduced

$$
\begin{aligned}
\tilde{Q}_{f} & =\left[\begin{array}{cc}
Q_{f} & L_{f}^{T} \\
L_{f} & 0
\end{array}\right], & \tilde{Q}_{d} & =\left[\begin{array}{cc}
Q_{d} & L_{d}^{T} \\
L_{d} & 0
\end{array}\right], \\
Q_{f} & =M_{f}^{T} M_{f}, & Q_{d} & =M_{d}^{T} M_{d}, \\
L_{f} & =N_{f}^{T} M_{f}, & L_{d} & =N_{d}^{T} M_{d}, \\
\tilde{c}_{f} & =N_{f}^{T} N_{f}, & \tilde{c}_{d} & =N_{d}^{T} N_{d} .
\end{aligned}
$$

It can be seen from (25) that those matrices with subscript $f$ are related to the effect of the fault while those matrices with subscript $d$ are related to the influence of the disturbance. Then, the FD optimization criterion (19) given in Proposition 5.2, which is obtained to describe the maximization of the effect of the faults (numerator) with respect to the effect of the disturbances (denominator), can be rewritten (simplified) using (25) as

$$
J(\tilde{\omega})=\frac{\tilde{\omega}^{T} \tilde{Q}_{f} \tilde{\omega}+\tilde{c}_{f}}{\tilde{\omega}^{T} \tilde{Q}_{d} \tilde{\omega}+\tilde{c}_{d}},
$$

where $\tilde{\omega}=\left[\begin{array}{c}\omega \\ 1\end{array}\right]$. The criterion $[26$ is a ratio of two quadratic functions. Apart from the constant terms in 26, the strong formal analogy with the design of parity space residuals using approximate decoupling techniques [46] can be observed. Though similar mathematical techniques can be used to solve the related optimization as detailed hereafter in Theorem 5.2 the purpose followed in this work is significantly different since it consists in obtaining a time-varying update of the gain of a state-bounding observer with a Kalman-like structure.

Theorem 5.2. (Optimal observer gain with FD purposes) Maximizing the criterion (18) and, equivalently, (26) means finding $\tilde{\omega}^{*}$ such that $J\left(\tilde{\omega}^{*}\right)=\max _{\tilde{\omega}} J(\tilde{\omega})$. The solution satisfies $\tilde{\omega}^{*} \in \operatorname{ker}\left(\tilde{Q}_{f}-J^{*} \tilde{Q}_{d}\right)$ and the optimal observer gain $G^{*}$ is determined by reshaping the solution of the generalized eigenvectors related to the greatest generalized eigenvalue of the pair $\left(\tilde{Q}_{f}, \tilde{Q}_{d}\right)$.

Proof. Consider Proposition 5.2 and select $\tilde{\omega}^{*}$ to be the optimal value of $\tilde{\omega}: \frac{\partial J(\tilde{\omega})}{\partial \tilde{\omega}}=0$. Hence, taking the derivative of 26] with respect to $\tilde{\omega}$ and setting it to zero successively yields

$$
\begin{aligned}
\tilde{\omega}^{T} \tilde{Q}_{f} \tilde{\omega}+\tilde{c}_{f}-J \tilde{\omega}^{T} \tilde{Q}_{d} \tilde{\omega}-J \tilde{c}_{d}=0, \\
2 \tilde{\omega}^{T} \tilde{Q}_{f}-J 2 \tilde{\omega}^{T} \tilde{Q}_{d}=0, \\
\left(\tilde{Q}_{f}-J^{*} \tilde{Q}_{d}\right) \tilde{\omega}^{*}=0,
\end{aligned}
$$

where the symmetric nature of $\tilde{Q}_{f}$ and $\tilde{Q}_{d}$ is taken into account. Therefore, a non-null solution satisfies

$$
\tilde{\omega}^{*} \in \operatorname{ker}\left(\tilde{Q}_{f}-J^{*} \tilde{Q}_{d}\right) .
$$

Thus, finding $\tilde{\omega}^{*}$ that maximizes $J$ can be achieved by computing the generalized eigenvalues and the related eigenvectors of the pair $\left(\tilde{Q}_{f}, \tilde{Q}_{d}\right)$. More precisely, the maximal value $J^{*}$ of $J$ for which a non-null solution exists is the greatest generalized eigenvalue of $\left(\tilde{Q}_{f}, \tilde{Q}_{d}\right)$ since the solutions $J$ to $\operatorname{det}\left(\tilde{Q}_{f}-J \tilde{Q}_{d}\right)=0$ ensure a non-zero kernel $\operatorname{ker}\left(\tilde{Q}_{f}-J \tilde{Q}_{d}\right)$. Then, the generalized eigenvector related to $J^{*}$ gives $\tilde{\omega}^{*}$. Finally, the optimal observer gain matrix is calculated by reshaping $\omega^{*}$ into a matrix as $G^{*}=\operatorname{col}^{-1}\left(\omega^{*}\right)$.

The statement and proof of Theorem 5.2 provide the core results of this paper leading to Algorithm 2 which implements the computation of the optimal observer gain for the proposed FD-ZKF FD filter.

A graphical representation of the proposed methodology using an observer gain $G$ (based on the ZKF approach) and the optimal observer gain $G^{*}$ (using the proposed FD-ZKF approach) for designing the state-bounding observer is presented in Figure 2. The image of unit hypercubes modeling the range of the possible disturbances and faults 


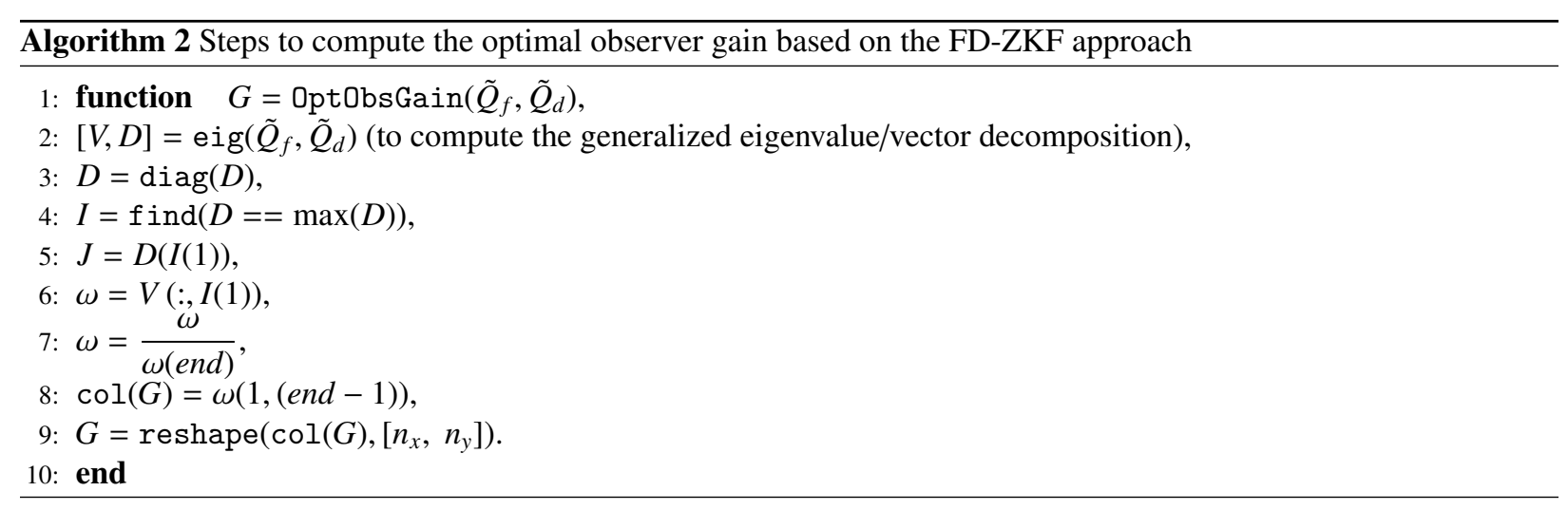

are illustrated by gray and empty zonotopes, respectively. Then, considering the same magnitude and direction of the fault $f$ in both cases, the detection of the fault cannot be guaranteed with the gain $G$ (see Figure 2 a). Indeed, Figure $2 \mathrm{a}$ illustrates a limit case between detection and non-detection: the influence of the fault exactly compensates that of the worst case disturbance, i.e., the red point is on the border of $R_{d}$. However, in Figure $2 \mathrm{p}$, the influence of the fault is far outside from the influence of disturbances. Therefore, the detection of the fault with the same fault magnitude (and even smaller ones) can be guaranteed only with $G^{*}$, i.e., when the observer gain results from the optimization of an FD criterion. The richer underlying set-based representation compared to a basic vector-norm approach gives additional degrees of freedom to deal with relative directions and bounds in the space characterizing the influence of the possible disturbance and fault signals.

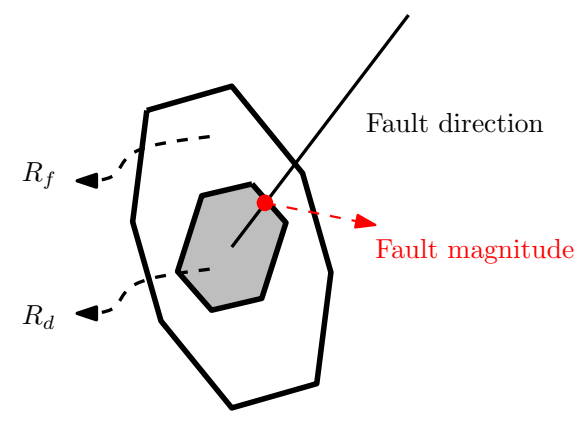

a) The case of using $G$

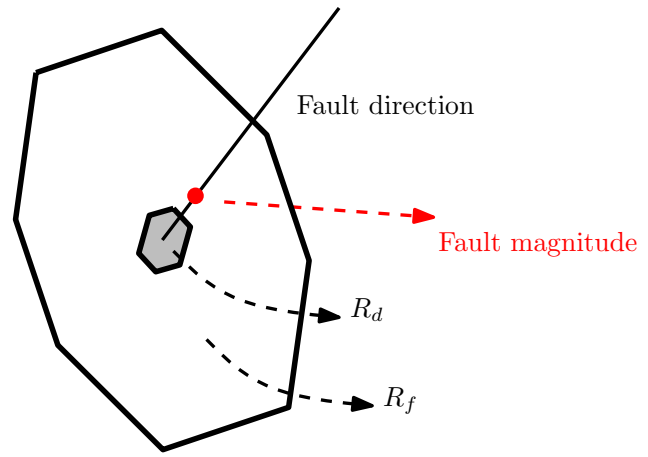

b) The case of using $G^{*}$

Figure 2: Intuitive graphical representation of the proposed method by means of plane zonotopes.

\section{Comparative assessment}

In this section, a comparative assessment of ZKF which optimizes the state observation criterion (16) and FD-ZKF which optimizes the FD criterion (18) is presented. To that purpose, the Minimum Detectable Fault (MDF) will be characterized based on a classical sensitivity analysis in order to show the effect of the observer gain over the MDF. Then, it is possible to compare the approaches using the mathematical expression of the MDF based on the model of a numerical example (which will be discussed in Section 7 .

Directly evaluating the MDF through simulations is an alternative way to show the improvement given by FD-ZKF compared to ZKF. A model-based analysis/characterization of the sensitivity to the faults is proposed in the following of this section. Here, the MDF is the minimum abrupt (step) fault that can be surely detected in steady state. For the sake of comparison only, it is assumed that the system is Linear Time-Invariant (LTI), whereas the general problem formulation of ZKF and FD-ZKF is still valid for LTV systems. 
In order to characterize the minimum magnitude of the fault that can be detected, the input-output form of the discrete-time dynamical model (3) is expressed as

$$
y(z)=\mathscr{T}_{u}(z) u(z)+\mathscr{T}_{d}(z) d(z)+\mathscr{T}_{f}(z) f(z),
$$

where the transfer function $\mathscr{T}(z)$ can be computed as

$$
\mathscr{T}(z)=C(z I-A)^{-1} B+D .
$$

Notice that the related input, disturbance and fault are denoted by the subscripts of $\mathscr{T}(z), B$ and $D$ as $u, d$ and $f$, respectively. The subscripts $u, d$ and $f$ are eliminated in (30) for the sake of simplified notations. Alternatively, considering (4) rather than (3), the measurement equation can also be expressed as

$$
y(z)=\mathscr{H}_{u}(z) u(z)+\mathscr{H}_{d}(z) d(z)+\mathscr{H}_{f}(z) f(z)+\mathscr{H}_{y}(z) y(z)
$$

with

$$
\begin{aligned}
& \mathscr{H}_{\bullet}(z)=C(z I-(A-G C))^{-1}\left(B_{\bullet}-G D_{\bullet}\right)+D_{\bullet}, \\
& \mathscr{H}_{y}(z)=C(z I-(A-G C))^{-1} G
\end{aligned}
$$

where subscript $\bullet$ can be assigned by $u, d$ and $f$ depending on the kind of input considered. Furthermore, the equation (31) can be written as

$$
\left(I-\mathscr{H}_{y}(z)\right) y(z)=\mathscr{H}_{u}(z) u(z)+\mathscr{H}_{d}(z) d(z)+\mathscr{H}_{f}(z) f(z)
$$

Therefore, the input-output form of the residual/innovation term can be written using (33) as

$$
\begin{aligned}
\varepsilon(z)=r(z) & =\left(I-\mathscr{H}_{y}(z)\right) y(z)-\mathscr{H}_{u}(z) u(z) \\
& =\underbrace{\mathscr{H}_{d}(z) d(z)}_{\varepsilon_{d}(z)}+\underbrace{\mathscr{H}_{f}(z) f(z)}_{\varepsilon_{f}(z)},
\end{aligned}
$$

where $\varepsilon_{d}(z)$ and $\varepsilon_{f}(z)$ refer to the (observer gain dependent) effect of the disturbance and the fault on the residual term. Thus, $\mathscr{H}_{d}(z)$ and $\mathscr{H}_{f}(z)$ can be interpreted as the sensitivity of the residual with respect to disturbances and faults, respectively.

Theorem 6.1. Given an observer as (5) and the input-output form of its measurement equation as (31), the Minimum Detectable Fault (MDF) in steady state is given under the single-fault assumption as

$$
\underline{f}_{j}^{\infty}=\min _{i} \underline{f}_{j i}^{\infty}, \quad \underline{f}_{j i}^{\infty}=2 \frac{\left\|\mathscr{H}_{d, i}(1)\right\|_{1}}{\left\|\mathscr{H}_{f, i j}(1)\right\|_{1}},
$$

where the magnitude $f_{j}$ of the single step faults that are necessarily detected satisfy $f_{j}>\underline{f}_{j}^{\infty}$ or $f_{j}<-\underline{f}_{j}^{\infty}$, with $j=1, \ldots, n_{f}$. A fault is said to be necessarily detected if $\exists k, 0 \notin\left\langle c_{\varepsilon}, b\left(R_{\varepsilon}\right)\right\rangle$ is satisfied. Whereas $j$ is an index for single faults, $i$ refers to the rows of $\mathscr{H}_{d}(z)$ and $\mathscr{H}_{f}(z)$. More precisely, $\mathscr{H}_{d, i}(1)$ is the $i^{\text {th }}$ row of $\mathscr{H}_{d}(1)$ and $\mathscr{H}_{f, i j}(1)$ is the element at the $i^{\text {th }}$ row and $j^{\text {th }}$ column of $\mathscr{H}_{f}(1)$.

Proof. Since $\varepsilon(z)=\varepsilon_{d}(z)+\varepsilon_{f}(z)$ in $z$-domain, the residual can be expressed in the time domain and in steady state (limit as $k \rightarrow \infty$ ) as

$$
\varepsilon^{\infty}=\varepsilon_{d}^{\infty}+\varepsilon_{f}^{\infty}=\mathscr{H}_{d}(1) d^{\infty}+\mathscr{H}_{f}(1) f^{\infty} .
$$

Considering $d \in[-1,1]^{n_{d}}=\left\langle 0, I_{n_{d}}\right\rangle$ and a faultless scenario (it means $f(z)=0 \Rightarrow f^{\infty}=0$ ), the residual in steady state can be expressed according to 36 as

$$
\varepsilon^{\infty} \in 0 \pm\left|\mathscr{H}_{d}(1)\right| \mathbf{1}
$$

where $|\cdot|$ is the element-by-element absolute value operator and $\mathbf{1}$ is a column vector of ones of appropriate dimension. Hence, $\left|\mathscr{H}_{d}(1)\right| \mathbf{1}$ is a vector whose $i^{\text {th }}$ element is the 1 -norm (scalar) of the $i^{\text {th }}$ row $\mathscr{H}_{d, i}(1)$ of the matrix $\mathscr{H}_{d}(1)$. Thus, 


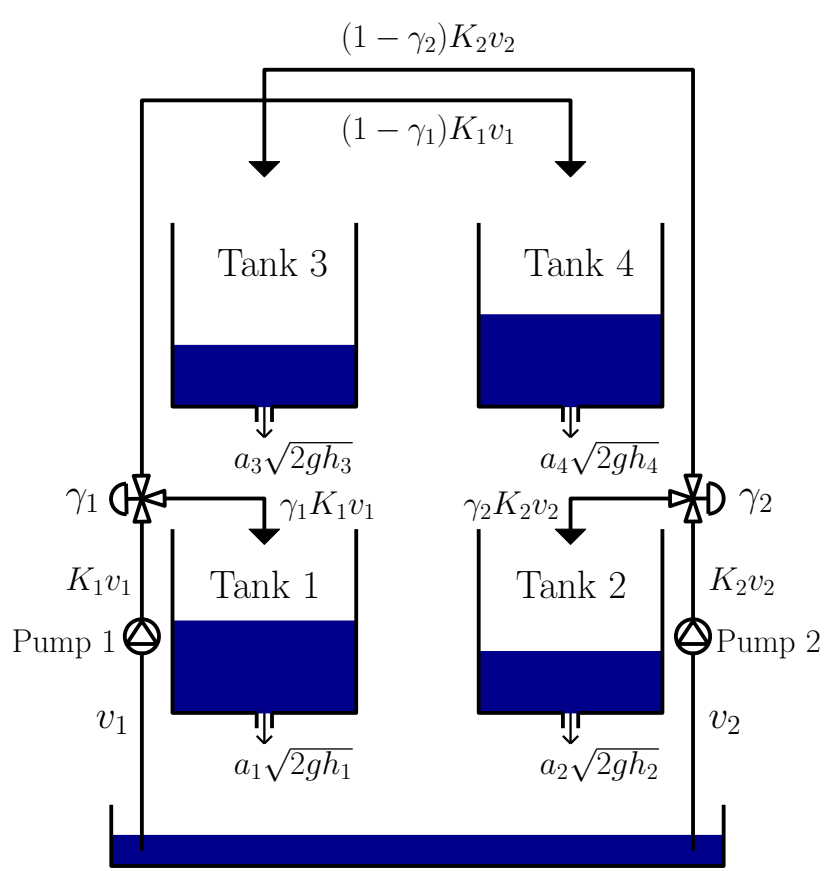

Figure 3: Schematic diagram of the quadruple-tank system

the $i^{\text {th }}$ element $\varepsilon_{i}^{\infty}$ of the residual $\varepsilon^{\infty}$ allows the detection of a fault if $\varepsilon_{i}^{\infty} \notin 0 \pm\left\|\mathscr{H}_{d, i}(1)\right\|_{1}$. Furthermore, (36) can be rewritten $\forall i$ as $\varepsilon_{i}^{\infty}=\varepsilon_{d, i}^{\infty}+\varepsilon_{f, i}^{\infty}$.

Therefore, in steady state, the condition ensuring the detection of the $j^{\text {th }}$ fault from the $i^{\text {th }}$ component of the residual is:

$$
\mathscr{H}_{f, i j}(1) f_{j}^{\infty} \notin 0 \pm 2\left\|\mathscr{H}_{d, i}(1)\right\|_{1} .
$$

The factor 2 comes from the worst-case scenario where the disturbances have a maximal influence in the opposite direction compared to that of the occurring fault. Thus, it can be written that

$$
f_{j}^{\infty} \notin 0 \pm 2 \frac{\left\|\mathscr{H}_{d, i}(1)\right\|_{1}}{\left\|\mathscr{H}_{f, i j}(1)\right\|_{1}} .
$$

Equation (38) can be rewritten as (39) so that $f_{j i}^{\infty}$ in 35 interprets as the minimum magnitude $f_{j}$ such that the $j^{\text {th }}$ fault is necessarily detected by the $i^{\text {th }}$ scalar residual $\varepsilon_{i}^{\infty}$ taken alone. This results in (35) when considering all the scalar components of the innovation term.

Finally, the comparison of the ZKF and FD-ZKF approaches can be done in the FD framework using Theorem 6.1. As it can be seen from (32), the transfer functions $\mathscr{H}_{d}$ and $\mathscr{H}_{f}$ in 35 depend on the observer gain. In the ZKF approach, the observer gain can be computed explicitly based on Section 5.2 using Theorem 5.1. In the FD-ZKF approach, the observer gain can be computed based on Section 5.3 using Algorithm 2 Because of their distinct observer gains, the FD performance is expected to be different between the ZKF and FD-ZKF approaches. In this regard, further quantitative comparison of the approaches will be discussed in Section 7 based on a case study.

\section{Case study}

\subsection{General description}

A case study based on a quadruple-tank system is used to illustrate the approach proposed in the previous sections. The quadruple tank is a multi input/multi output process proposed by [47]. Furthermore, the system contains two 
Table 1: Model parameters.

\begin{tabular}{|ll|}
\hline Parameter value & Unit \\
\hline \hline$A_{1}=A_{3}=28$, & $\mathrm{cm}^{2}$ \\
$A_{2}=A_{4}=32$ & $\mathrm{~cm}^{2}$ \\
$a_{1}=a_{3}=0.071$ & $\mathrm{~cm}^{2}$ \\
$a_{2}=a_{4}=0.057$ & $\mathrm{~cm}^{2}$ \\
$g=981$ & $\mathrm{~cm} / \mathrm{s}^{2}$ \\
\hline
\end{tabular}

Table 2: Value of variables.

\begin{tabular}{|ccc|}
\hline operating point & Parameter value & Unit \\
\hline \hline$\left(h_{1}^{*}, h_{2}^{*}\right)$ & $(12.4,12.7)$ & $\mathrm{cm}$ \\
$\left(h_{3} *, h_{4}^{*}\right)$ & $(1.8,1.4)$ & $\mathrm{cm}$ \\
$\left(v_{1} *, v_{2}^{*}\right)$ & $(3,3)$ & $\mathrm{V}$ \\
$\left(K_{1} *, K_{2}^{*}\right)$ & $(3.33,3.35)$ & $\mathrm{cm}^{3} / \mathrm{Vs}$ \\
$\left(\gamma_{1} *, \gamma_{2}^{*}\right)$ & $(0.7,0.6)$ & - \\
\hline
\end{tabular}

pumps and four interconnected tanks.

As it can be seen from the schematic diagram of the system setup in Figure 3, the two process inputs are the pump flows that are determined by the voltages $v_{1}$ and $v_{2}$ (input voltages to the pumps varying between $0 \mathrm{~V}$ to $10 \mathrm{~V}$ ). Furthermore, the outputs of the process are the water levels in the lower tanks that are obtained as voltages from the measurement devices with the range between $0 \mathrm{~V}$ to $10 \mathrm{~V}$. Tanks 3 and 4 are placed on top of Tanks 1 and 2 . In addition, the action of pumps is to fill the tanks by extracting the water from the basin. Furthermore, Tanks 1 and 2 receive additional water flow from Tanks 3 and 4 because of the gravity effect. The water flow to each tank is controlled by the position of the valves determined by $\gamma_{1}$ and $\gamma_{2}$ in Figure 3 Furthermore, the position of the valves $\gamma_{1}, \gamma_{2} \in(0,1)$ are the ratios modeling how the output of the pumps are divided between the upper and lower tanks.

Regarding the physical features, the height of each tank is $20 \mathrm{~cm}$ and the connection of the tank and the pump is done using a pipe with a diameter equal to $6 \mathrm{~mm}$.

The mathematical model of the process can be determined based on the mass balance relations and Bernoulli's law as

$$
\begin{aligned}
& \frac{d h_{1}(t)}{d t}=-\frac{a_{1}}{A_{1}} \sqrt{2 g h_{1}(t)}+\frac{a_{3}}{A_{1}} \sqrt{2 g h_{3}(t)}+\frac{\gamma_{1} K_{1}}{A_{1}} v_{1}(t), \\
& \frac{d h_{2}(t)}{d t}=-\frac{a_{2}}{A_{2}} \sqrt{2 g h_{2}(t)}+\frac{a_{4}}{A_{2}} \sqrt{2 g h_{4}(t)}+\frac{\gamma_{2} K_{2}}{A_{2}} v_{2}(t), \\
& \frac{d h_{3}(t)}{d t}=-\frac{a_{3}}{A_{3}} \sqrt{2 g h_{3}(t)}+\frac{\left(1-\gamma_{2}\right) K_{2}}{A_{3}} v_{2}(t), \\
& \frac{d h_{4}(t)}{d t}=-\frac{a_{4}}{A_{4}} \sqrt{2 g h_{4}(t)}+\frac{\left(1-\gamma_{1}\right) K_{1}}{A_{4}} v_{1}(t),
\end{aligned}
$$

where

- $h_{i}$ with $i=1,2,3,4$ is the water level in Tank $i$,

- $A_{i}$ is the cross section of Tank $i$ with $i=1,2,3,4$,

- $a_{i}$ is the cross section of the outlet pipe with $i=1,2,3,4$,

- $K_{j}$ is the constant of the Pump $j$ with $j=1,2$,

- $g$ is the acceleration due to gravity.

The value of the parameters of (40) is presented in Table 1 . To apply the approach proposed in this paper, the non-linear model (40) is linearized around the working point that is presented using the superscript $*$ in Table 2 and introducing the variables $\tilde{h}_{i}=h_{i}-h_{i}^{*}$ and $\tilde{v}_{i}=v_{i}-v_{i}^{*}$ as 


$$
\begin{aligned}
& \dot{\tilde{h}}(t)=\left[\begin{array}{cccc}
-\frac{1}{T_{1}} & 0 & \frac{A_{3}}{A_{1} T_{3}} & 0 \\
0 & -\frac{1}{T_{2}} & 0 & \frac{A_{4}}{A_{2} T_{4}} \\
0 & 0 & -\frac{1}{T_{3}} & 0 \\
0 & 0 & 0 & -\frac{1}{T_{4}}
\end{array}\right] \tilde{h}(t)+\left[\begin{array}{cc}
\frac{\gamma_{1} K_{1}}{A_{1}} & 0 \\
0 & \frac{\gamma_{2} K_{2}}{A_{2}} \\
0 & \frac{\left(1-\gamma_{1}\right) K_{2}}{A_{3}} \\
\frac{\left(1-\gamma_{1}\right) K_{1}}{A_{4}} & 0
\end{array}\right] \tilde{v}(t), \\
& y(t)=\left[\begin{array}{cccc}
K_{c} & 0 & 0 & 0 \\
0 & K_{c} & 0 & 0
\end{array}\right] \tilde{h}(t),
\end{aligned}
$$

where the measured level signals are obtained considering that $K_{c}=0.5 \mathrm{~V} / \mathrm{cm}$ and $T_{i}=\frac{A_{i}}{a_{i}} \sqrt{\frac{2 h_{i}^{*}}{g}}$, with $i=1,2,3,4$ as $T_{1}=62.7034, T_{2}=90.3353, T_{3}=23.8900$ and $T_{4}=29.9930$.

Finally, using the Euler discretization with a sampling time of 1s, a discrete-time linear model is obtained as

$$
\begin{gathered}
\tilde{h}_{+}=A \tilde{h}+B_{u} \tilde{v}+B_{d} d+B_{f} f, \\
y=C \tilde{h}+D_{u} \tilde{v}+D_{d} d+D_{f} f,
\end{gathered}
$$

with

$$
\begin{array}{rlrl}
A & =\left[\begin{array}{cccc}
0.9841 & 0 & 0.0419 & 0 \\
0 & 0.9889 & 0 & 0.0333 \\
0 & 0 & 0.9581 & 0 \\
0 & 0 & 0 & 0.9667
\end{array}\right], B_{u}=\left[\begin{array}{cc}
0.2102 & 0 \\
0 & 0.0628 \\
0 & 0.0479 \\
0.0094 & 0
\end{array}\right], \\
C=\left[\begin{array}{cccc}
0.5 & 0 & 0 & 0 \\
0 & 0.5 & 0 & 0
\end{array}\right], & & & D_{u}=\left[\begin{array}{ll}
0 & 0 \\
0 & 0
\end{array}\right] .
\end{array}
$$

Taking into account the state disturbance and measurement noise, $B_{d} d$ and $D_{d} d$ are used in (42) with

$$
\begin{aligned}
B_{d} & =\left[\begin{array}{ccccccc}
0.05 & 0.01 & 0 & 0 & 0 & 0 & 0 \\
0.05 & 0 & 0.01 & 0 & 0 & 0 & 0 \\
0.05 & 0 & 0 & 0.01 & 0 & 0 & 0 \\
0.05 & 0 & 0 & 0 & 0.01 & 0 & 0
\end{array}\right], \\
D_{d} & =\left[\begin{array}{lll}
02 x 5 & 0.1 & I_{2}
\end{array}\right],
\end{aligned}
$$

As it can be seen in (44a), the first column of $B_{d}$ is used to define a disturbance influencing all the states, e.g., rain simultaneously getting into all the tanks. The idea of considering the rain that can have large influence on the direction defined by the first column of matrix $B_{d}$ in (44a) is to model a kind of flow (disturbance) affecting all tanks at the same time. In addition, disturbances aiming at enclosing modeling errors like, e.g., linearization and discretization errors, are introduced through the next diagonal block in $B_{d}$. Therefore, bounded disturbances acting in all the state-space directions and the measurement noise are modeled with $B_{d}$ and $D_{d}$, respectively.

Furthermore, the effect of faults on the state and the measurements is modeled through the terms $B_{f} f$ and $D_{f} f$, respectively. The possible faults that are considered are actuator faults, sensor faults and leakages. Hence, the following matrices are chosen in the simulation in order to simulate all these faults:

$$
\begin{aligned}
B_{f} & =\left[\begin{array}{lll}
10 B_{u} & 0.3 I_{4} & 0_{4 x 2}
\end{array}\right], \\
D_{f} & =\left[\begin{array}{lll}
0_{2 \times 6} & 10 I_{2}
\end{array}\right] .
\end{aligned}
$$

Consistently with the problem formulation in Section 3 , the uncertain input vectors $d$ and $f$ are assumed to be normalized in $[-1,+1]$. The values of $B_{d}, D_{d}, B_{f}$ and $D_{f}$ are defined accordingly. Then, a $10 \%$ step fault $f_{i}$ is 

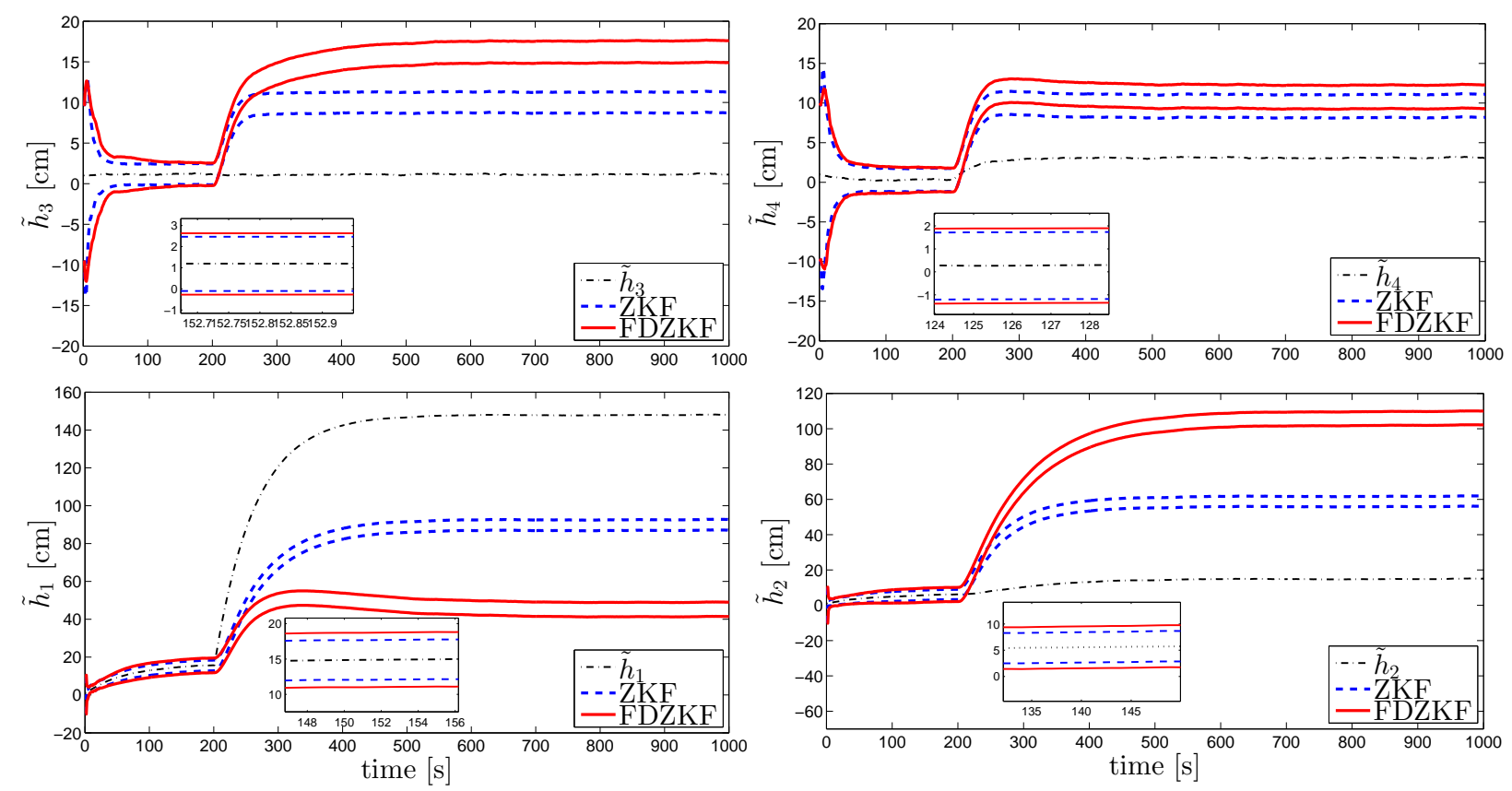

Figure 4: State estimation in the case of an actuator fault $f_{1}$

simulated with $f_{i}=0.1$ after the fault occurrence.

\subsection{FD-ZKF filter implementation}

The observer gain can be iteratively computed for the quadruple-tank system presented in (42) using Theorem 5.1 in the case of ZKF, and Algorithm 2 in the case of FD-ZKF. In both cases, the FD test is implemented based on Algorithm 1 .

\subsection{Performing $F D$}

Two FD tests are considered in this section. Both are implemented using the Algorithm 1 . They only differ from the observer gain used: it is determined using the ZKF or FD-ZKF approach as explained in the Section5.

Regarding the disturbance scenario, (44) is used to simulate all the possible disturbances acting in all the directions. Furthermore, regarding the fault simulation, the following fault scenario is set in all the simulations: from time instants $k=0$ to $k=200$, the system is healthy. Then, an additive step (abrupt) fault occurs at time instant $k=200$ and it remains in the system until $k=1000$. Moreover, single faults are considered based on the elements of the vector $f$, i.e.,

$$
f=\left[\begin{array}{llllllll}
f_{1} & f_{2} & f_{3} & f_{4} & f_{5} & f_{6} & f_{7} & f_{8}
\end{array}\right]^{T},
$$

where $f_{1}$ and $f_{2}$ indicate the actuator faults, $f_{3}, f_{4}, f_{5}$ and $f_{6}$ are the leakage faults and the sensor faults are denoted by $f_{7}$ and $f_{8}$.

In the first simulation, the FD test is done when considering the actuator fault $f_{1}$, i.e, the faultless scenario is considered from the beginning of the simulation until time instant $k=200$. Then, the occurrence of the actuator fault $f_{1}$ is simulated at $k=200$ and it remains until the end of the simulation. Figure 4 shows the projection of the computed state-bounding zonotope into the state space when the actuator fault occurs at time instant $k=200$. The state estimation in this figure is carried out by considering Proposition 4.1 using ZKF and FD-ZKF approaches to compute the observer gain. As it was mentioned before, the fault is simulated after time instant $k=200$. Thus, before this time instant, the system is only affected by the effect of disturbances and noises. Consequently, the bounds that are obtained at the first 200 time instants show the effect of both disturbances and noises. As it can be seen from the 


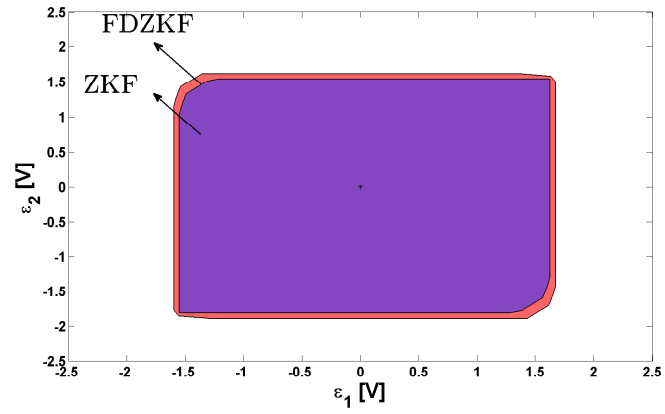

Figure 5: Zonotopes bounding the innovation/residual before the occurrence of the fault at $k=100$

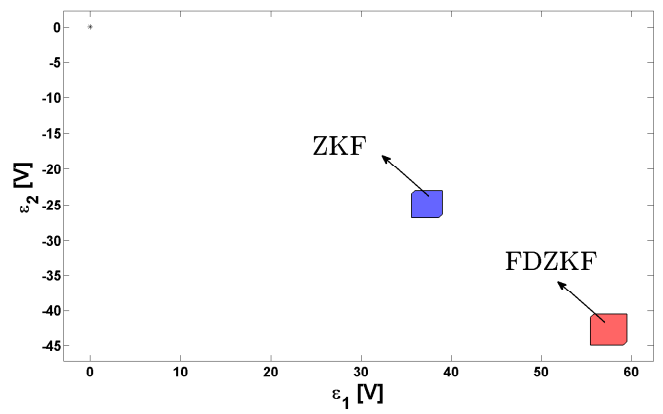

Figure 6: Zonotopes bounding the innovation/residual after the occurrence of the fault at $k=600$

time instant $k=0$ until $k=200$ where the system is only affected by the disturbance, the observer can properly follow the system using both observer gains.

Furthermore, Figure 4 shows that the state estimation bounds are a bit more conservative with FD-ZKF compared to ZKF for $0 \leq k<200$. A possible explanation is related to the criteria of the approaches. The ZKF optimization criterion is defined considering only the observation purposes whereas the FD-ZKF criterion is defined considering FD performance. Thus, it is normal to obtain a better state estimation with ZKF. What is interesting in Figure 4 is that even by considering FD purpose with the FD-ZKF approach, no significant differences are obtained from the perspective of state estimation in comparison with ZKF.

After time instant $k=200$, the effect of the actuator fault can be seen on the state estimation in Figure 4 . The inconsistency of the observation by the model using both ZKF and FD-ZKF approaches and the nominal behaviour of the system allows to detect the fault. Furthermore, comparing the approaches after the fault occurrence reveals the improvement provided by the FD-ZKF approach since its estimation envelope has more changes with respect to the behaviour of the true system, so showing an increased sensitivity to the considered fault. Additionally, it can be observed that the improved sensitivity of the FD-ZKF approach is persistent, even after reaching the steady state.

For further illustration, the FD test is done based on Algorithm 1 considering the innovation term. As it is shown in
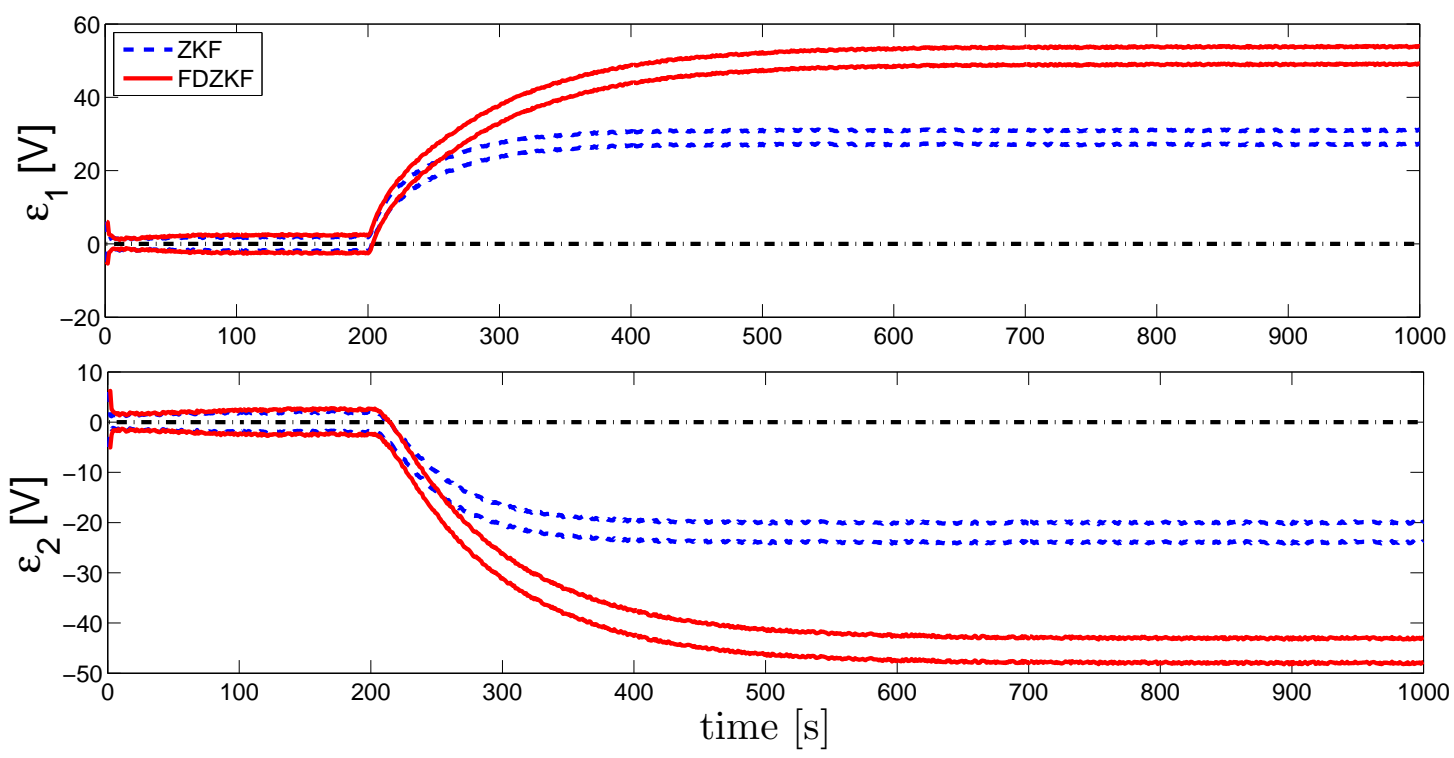

Figure 7: Envelopes of the scalar innovation terms 
Figure 5. a threshold (here zero) is included by both innovation zonotopes generated by ZKF and FD-ZKF approaches, respectively, before the occurrence of the fault. But, after the fault occurrence, both zonotopes move and the fault can be detected since zero is outside of the zonotopes bounding the innovation. The higher sensitivity of the FD-ZKF approach in comparison with ZKF approach can be seen in Figure 6 since its generated zonotope moves further from the non-faulty region. Therefore, it can be obsrved that FD-ZKF is more sensitive with respect to the effect of the fault.

Moreover, for the completion of the analysis, it is also interesting to combine the innovation terms and see the effect of the fault over both innovation terms together. In this regard, the ratio between the Euclidean norm of the center and the generator matrix of the innovation term is computed for both ZKF and FD-ZKF approaches. In particular, the ratio $\frac{\left\|c_{\varepsilon}\right\|_{2}}{\left\|R_{\varepsilon}\right\|_{2}}$ is compared when the state-bounding observer is designed using both approaches. Figure 8 shows the results obtained in this context.

As expected from the previous results in Figure 7, the most sensitive performance is obtained with FD-ZKF. But, now Figure 8 presents a single scalar criterion aggregating all the scalar components of the innovation that provides a well-defined basis for the comparison of the approaches. Indeed, directly comparing the values of the optimization criteria (16) and (18) is not relevant since they consider different goals. Moreover, considering independently each scalar term of the innovation as in Figure 7 may significantly complicate the sensitivity analysis when the number of sensors is greater than 2 or 3. Moreover, the bottom plot in Figure 8 shows the FD test decision resulting from both approaches: 0 means no-fault affecting the system and 1 means the fault is detected.

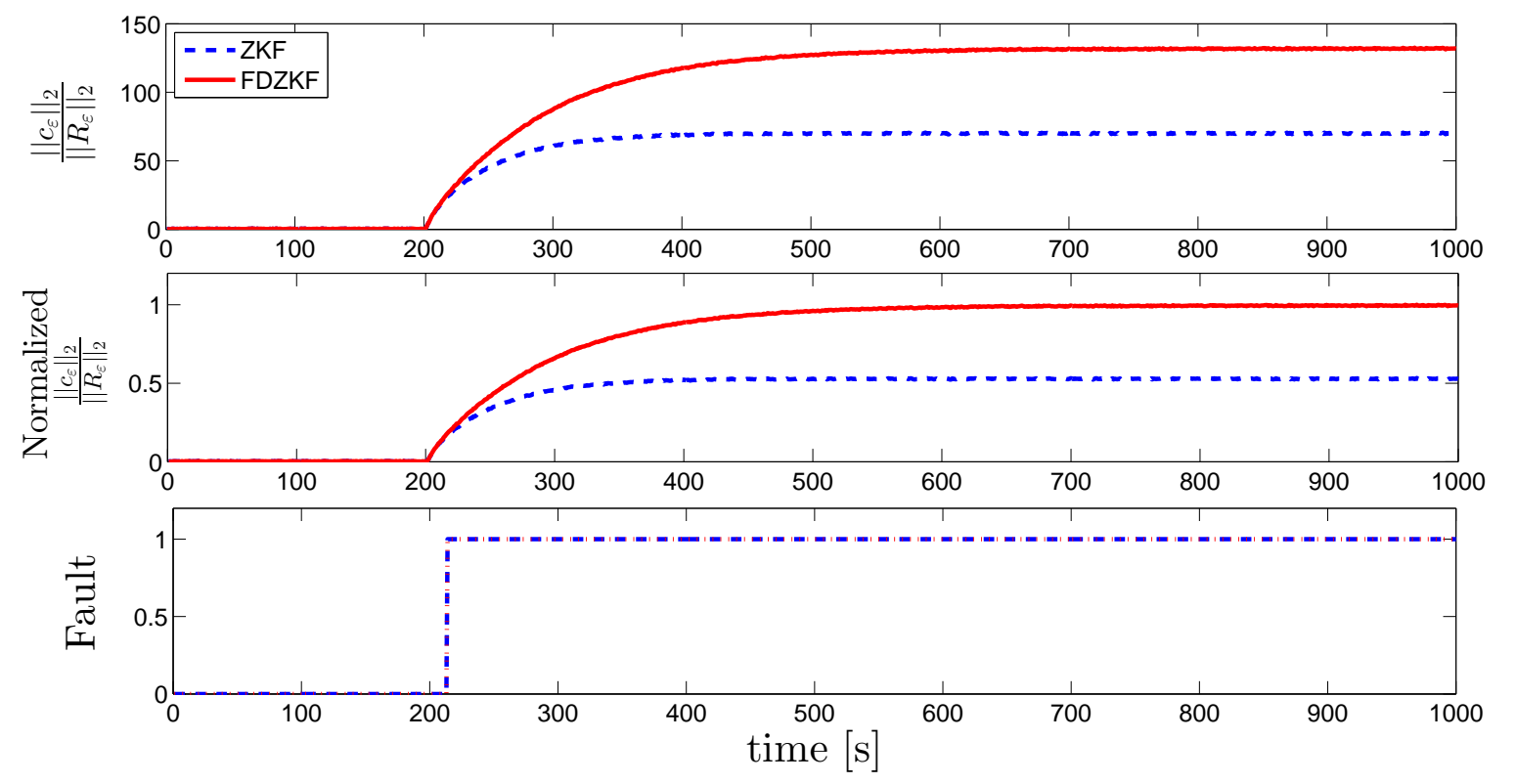

Figure 8: Ratio $\frac{\left\|c_{\varepsilon}\right\|_{2}}{\left\|R_{\varepsilon}\right\|_{2}}$ and the FD test in the case of an actuator fault $f_{1}$.

The second considered scenario corresponds to a sensor fault. The output of the system is measured from the level measurement device. Since the height of each tank is $20 \mathrm{~cm}$, the output of the system from the level measurement device is between $0-10 \mathrm{~V}$. The matrix $D_{f}$ in $45 \mathrm{~b}$ is defined with the whole range of the measurement. Then, the simulation of a step sensor fault with a magnitude of $10 \%$ of the whole range is simulated after the fault occurrence with

$$
f=\left[\begin{array}{llllllll}
0 & 0 & 0 & 0 & 0 & 0 & 0 & 0.1
\end{array}\right]^{T} .
$$

As in the case of the actuator fault, the ratio between the Euclidean norm of the center and the generator matrix of the innovation term is computed and reported in Figure 9 Once again, the sensitivity to the fault with FD-ZKF is improved compared to ZKF. 

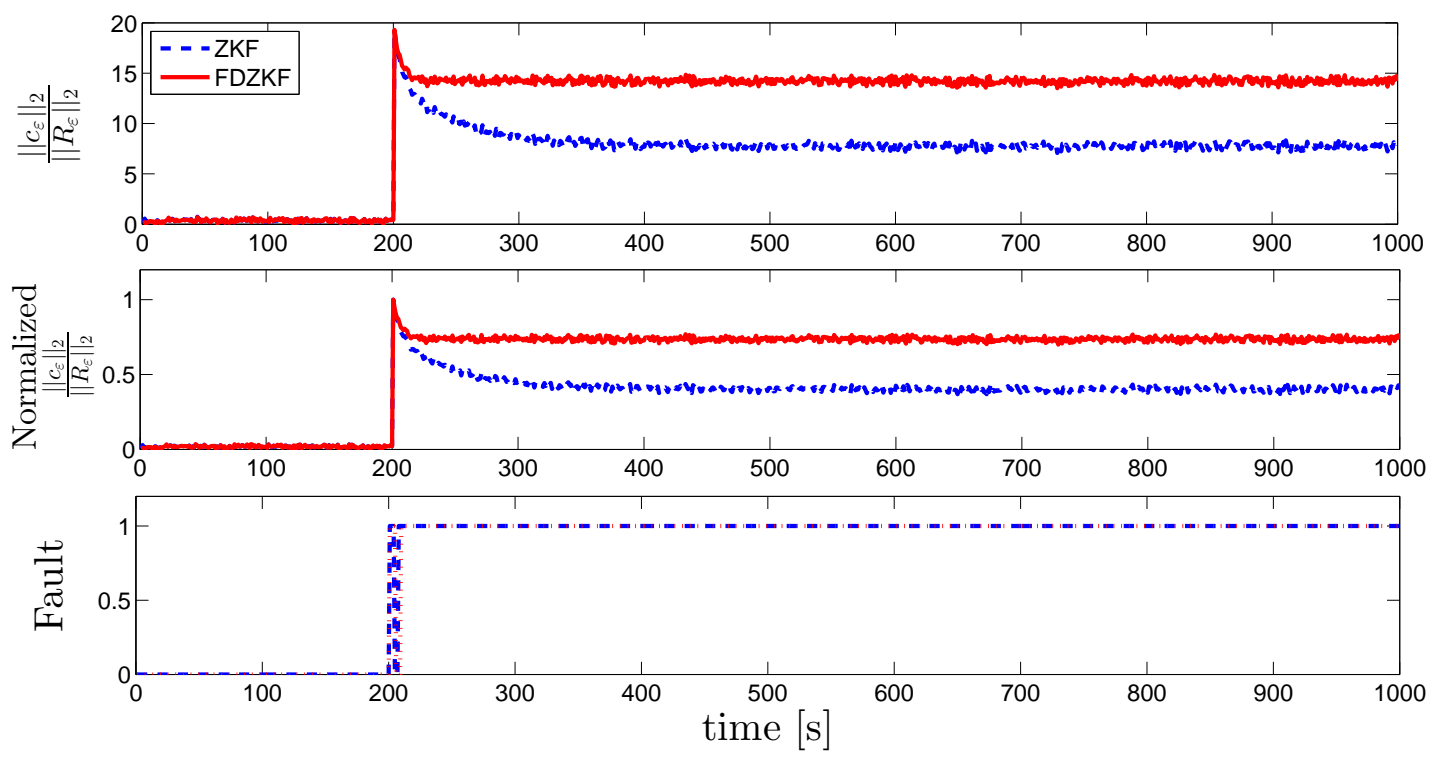

Figure 9: Ratio $\frac{\left\|c_{\varepsilon}\right\|_{2}}{\left\|R_{\varepsilon}\right\|_{2}}$ and the FD test in the case of a sensor fault $f_{8}$

The last scenario considered to test the proposed FD-ZKF approach corresponds to a leakage fault $f_{3}$ simulated using $B_{f}$ as in 45a). Figure 10 shows a similar improvement of the sensitivity with FD-ZKF and an analog detection ability compared to the other fault scenarios (sensor and actuator faults).

\subsection{Minimum Detectable Faults (MDF) analysis}

Based on Theorem 6.1, the computation of the MDF is influenced by the observer gain. In order to compute the MDF for the case study, (35) is used. Therefore, based on the different observer gains $G_{\infty}^{*}$ obtained with ZKF and FDZKF, magnitudes of the the MDF can be determined. Moreover, the constant observer gain that is obtained in steady state can be used to compute the MDF that can be detected in order to compare the performance of the observer when using FD-ZKF and ZKF approaches. In this regard, the following observer gains are obtained in steady state from the simulation of the two approaches:

$$
\begin{aligned}
\text { FD-ZKF approach: } & G_{\infty}^{*}=\left[\begin{array}{ll}
0.0679 & 0.0804 \\
0.0807 & 0.0746 \\
0.0469 & 0.0391 \\
0.0484 & 0.0469
\end{array}\right], \\
\text { ZKF approach: } & G_{\infty}^{*}=\left[\begin{array}{ll}
0.0828 & 0.0730 \\
0.0731 & 0.0845 \\
0.0446 & 0.0421 \\
0.0466 & 0.0475
\end{array}\right],
\end{aligned}
$$

where $G_{\infty}^{*}$ is the obtained optimal gain in steady state.

Then, the obtained results based on Theorem 6.1 are reported in Table 3 . This means that the detection of the fault with the magnitude either bigger than the obtained magnitude in Table 3 or smaller than the obtained magnitude in Table 3 with negative sign can be guaranteed. Furthermore, from Table 3 and for the considered faults, the size of the MDF is systematically smaller in case of the FD-ZKF. This illustrates the sensitivity improvement obtained by the proposed optimal tuning of the observer gain in a FD framework.

Further simulations are carried out for the case study by changing the fault magnitudes. It is interesting to see the magnitude of the faults that can be detected considering not only the steady state (Table 4), but also the whole time 

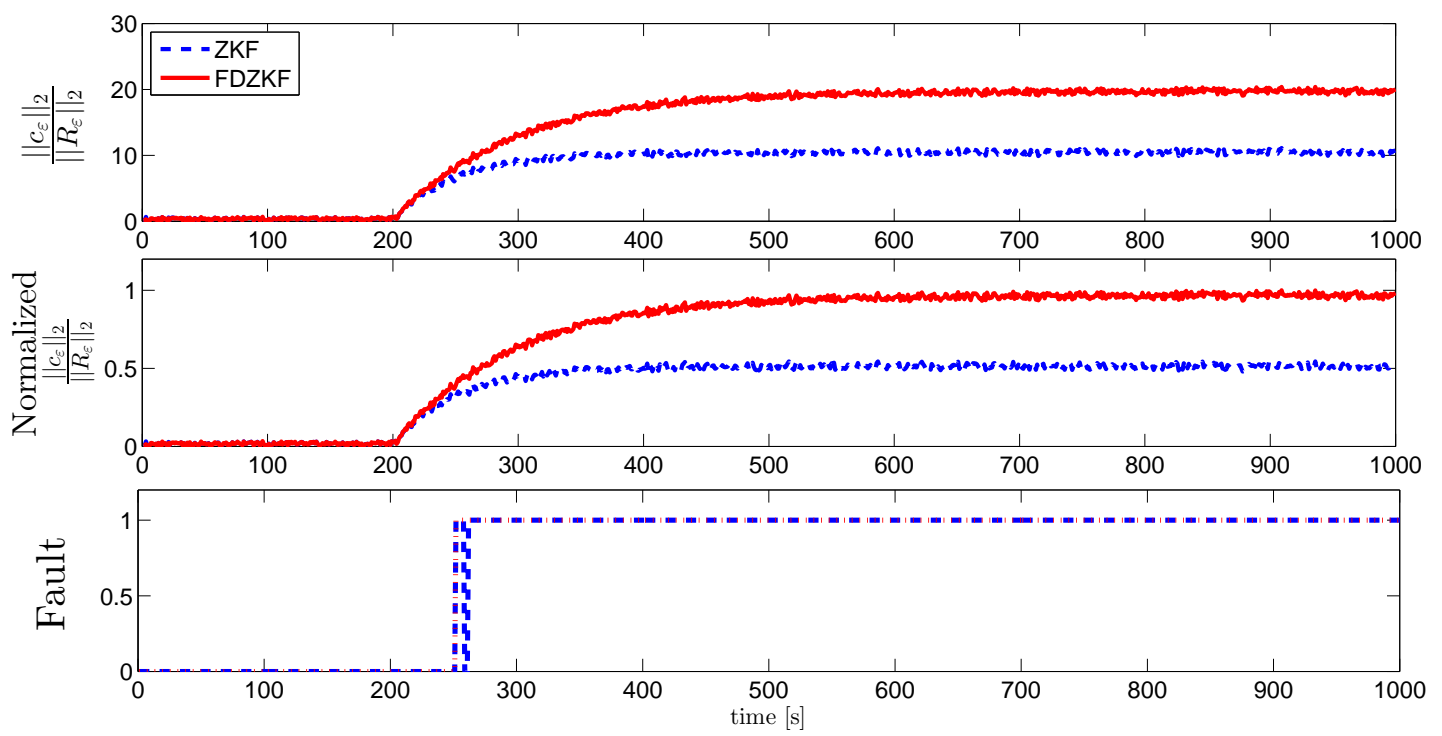

Figure 10: Ratio $\frac{\left\|c_{\varepsilon}\right\|_{2}}{\left\|R_{\varepsilon}\right\|_{2}}$ and the FD test in the case of a leakage fault $f_{3}$

range of the simulation as reported in Table 5. By increasing the magnitude of the fault, the FD-ZKF detection test (Algorithm 1) is used to see whether the fault with this magnitude can be detected or not. The results of this analysis are collected in Tables 4 and 5 .

Table 4 shows those magnitudes of the fault that can be still detected at the end of the simulation, i.e., in steady state. Therefore, data from this table can be compared with the data in Table 3, which shows the theoretical MDF magnitudes obtained from Theorem 6.1 In this regard, by comparing Tables 3 and 4 , no significant differences are found between the size of the MDF in all the cases. Hence, Table 4 confirms through numerical simulations the theoretical values previously reported in Table 3 and the improvement achieved by FD-ZKF. Further analysis is done in Table 5 to obtain the magnitude that can be detectable in whole the time range of the simulation.

Moreover, it can be observed from the comparison of Tables 4 and 5 , that the magnitude of the fault that can be detected considering the whole time range of the simulation is almost the same in the case of actuator and leakage fault. However, in the case of sensor faults and because of the fault reinjection involved by the observer structure leading to some transient behavior (see the overshoot in Figure 9p, the magnitude of the detectable fault is improved on the whole time range of the simulation compared to steady state only. This illustrates that FD-ZKF is well suited to also address time-varying and transient behaviors to enhance the FD ability.

Table 3: MDF (theoretical sensitivity analysis under a steady-state operation)

\begin{tabular}{l||cc|cccc|cc} 
& \multicolumn{2}{|c|}{ Actuator fault } & \multicolumn{4}{c|}{ Leakage fault } & \multicolumn{2}{c}{ Sensor fault } \\
& $f_{1}$ & $f_{2}$ & $f_{3}$ & $f_{4}$ & $f_{5}$ & $f_{6}$ & $f_{7}$ & $f_{8}$ \\
\hline \hline ZKF approach & 0.0641 & 0.5291 & 0.4340 & 0.4300 & 0.4340 & 0.4300 & 0.4082 & 0.5826 \\
\hline FD-ZKF approach & 0.0578 & 0.4812 & 0.3898 & 0.3641 & 0.3898 & 0.3641 & 0.3667 & 0.4934 \\
\hline Improvement & $9.8284 \%$ & $9.0531 \%$ & $10.1843 \%$ & $15.3256 \%$ & $10.1843 \%$ & $15.3256 \%$ & $10.1666 \%$ & $15.3107 \%$
\end{tabular}

\section{Conclusion}

This paper has proposed a new Fault Detection (FD) observer based on a Zonotopic Kalman Filter (ZKF), called FD-ZKF, that enhances the sensitivity to faults while increasing the robustness to disturbances. As a novelty, in the proposed FD-ZKF approach, the time-varying observer gain is optimized by considering the FD purposes and it can 
Table 4: MDF at the end of the simulation

\begin{tabular}{l||cc|cccc|cc}
\multicolumn{1}{l|}{} & \multicolumn{2}{c|}{ Actuator fault } & \multicolumn{4}{c|}{ Leakage fault } & \multicolumn{2}{c}{ Sensor fault } \\
& $f_{1}$ & $f_{2}$ & $f_{3}$ & $f_{4}$ & $f_{5}$ & $f_{6}$ & $f_{7}$ & $f_{8}$ \\
\hline \hline ZKF approach & 0.0472 & 0.5012 & 0.4132 & 0.4121 & 0.4122 & 0.4313 & 0.3990 & 0.5912 \\
\hline FD-ZKF approach & 0.0421 & 0.4620 & 0.3621 & 0.3412 & 0.3592 & 0.3552 & 0.3492 & 0.4897 \\
\hline Improvement & $10.8051 \%$ & $7.8212 \%$ & $12.3669 \%$ & $17.2046 \%$ & $12.8578 \%$ & $17.6443 \%$ & $12.4812 \%$ & $17.1685 \%$
\end{tabular}

Table 5: MDF by considering the whole time range of the simulation

\begin{tabular}{l||cc|cccc|cc}
\multicolumn{1}{c||}{} & \multicolumn{2}{c|}{ Actuator fault } & \multicolumn{4}{c|}{ Leakage fault } & \multicolumn{2}{c}{ Sensor fault } \\
& $f_{1}$ & $f_{2}$ & $f_{3}$ & $f_{4}$ & $f_{5}$ & $f_{6}$ & $f_{7}$ & $f_{8}$ \\
\hline \hline ZKF approach & 0.04901 & 0.4997 & 0.4139 & 0.4098 & 0.4134 & 0.4231 & 0.3582 & 0.5718 \\
\hline FD-ZKF approach & 0.0431 & 0.4631 & 0.3597 & 0.3396 & 0.3517 & 0.3497 & 0.3013 & 0.4698 \\
\hline Improvement & $12.0588 \%$ & $7.3244 \%$ & $13.0950 \%$ & $17.1303 \%$ & $14.9250 \%$ & $17.3481 \%$ & $15.8850 \%$ & $17.8384 \%$
\end{tabular}

be perceived as an extended version to FD of the ZKF approach where the observer gain is only computed for state observation purposes. In the proposed algorithm, the influences of all possible disturbances and faults within the specified ranges have been considered to compute the observer gain with the aim of increasing the sensitivity to faults with respect to disturbances. This is achieved through the optimization of a set-based criterion explicitly taking the relative influence of faults with respect to disturbances into account. Furthermore, the Minimum Detectable Fault (MDF) is characterized using a classical sensitivity analysis in order to show the effectiveness of the proposed timevarying observer gain on FD performance. The comparison of FD-ZKF and ZKF approaches has been conducted on a case study based on a quadruple-tank system. The obtained results show a significant improvement of the FD$\mathrm{ZKF}$ approach in comparison with the ZKF approach in FD performance. Furthermore, a quite small difference is obtained between the approaches when computing the state-bounding sets. Thus, a small relaxation of the state observation ability has given sufficient freedom degrees to significantly enhance the efficiency of FD. As a future research, enhancing the sensitivity to specific kind of faults will be considered to improve not only the fault detection performances, but also the fault isolation capabilities.

\section{Acknowledgments}

This work has been partially funded by the Spanish State Research Agency (AEI) and the European Regional Development Fund (ERFD) through the projects DEOCS (ref. MINECO DPI2016-76493) and SCAV (ref. MINECO DPI2017-88403-R). This work has also been partially funded by AGAUR of Generalitat de Catalunya through the Advanced Control Systems (SAC) group grant (2017 SGR 482) and by Agència de Gestió d'Ajuts Universitaris i de Recerca. Furthermore, the authors would like to thank Prof. A. Zolghadri for insightful discussions on ZKF extensions and applications within SysNum cluster activities (Idex Bordeaux, France).

\section{References}

[1] M. Blanke, M. Kinnaert, J. Lunze, M. Staroswiecki, J. Schröder, Diagnosis and fault-tolerant control, Vol. 691, Springer, 2006.

[2] J. Chen, R. J. Patton, Robust model-based fault diagnosis for dynamic systems, Vol. 3, Springer Science \& Business Media, 2012.

[3] V. Puig, S. Montes de Oca, J. Blesa, Adaptive threshold generation in robust fault detection using interval models: time-domain and frequencydomain approaches, International Journal of Adaptive Control and Signal Processing 27 (10) (2013) 873-901.

[4] V. Puig, Fault diagnosis and fault tolerant control using set-membership approaches: Application to real case studies, Applied Mathematics and Computer Science 20 (4) (2010) 619-635.

[5] T. Alamo, J. M. Bravo, E. F. Camacho, Guaranteed state estimation by zonotopes, Automatica 41 (6) (2005) $1035-1043$.

[6] T. Raïssi, D. Efimov, A. Zolghadri, Interval state estimation for a class of nonlinear systems, IEEE Transactions on Automatic Control 57 (1) (2012) 260-265.

[7] M. Pourasghar, V. Puig, C. Ocampo-Martinez, Q. Zhang, Reduced-order interval-observer design for dynamic systems with time-invariant uncertainty, IFAC-PapersOnLine 50 (1) (2017) 6271-6276.

[8] D. Efimov, T. Raïssi, A. Zolghadri, Control of nonlinear and LPV systems: interval observer-based framework, IEEE Transactions on Automatic Control 58 (3) (2013) 773-778. 
[9] F. Karimi Pour, V. Puig, C. Ocampo-Martínez, Comparative assessment of LPV-based predictive control strategies for a pasteurization plant, in: 4th International Conference on Control, Decision and Information Technologies, 2017, Spain, pp. 1-6.

[10] R. E. Kalman, A new approach to linear filtering and prediction problems, Journal of Basic Engineering 82 (1) (1960) 35-45.

[11] P. S. Maybeck, Stochastic models, estimation, and control, Vol. 3, Academic press, 1982.

[12] F. Schweppe, Recursive state estimation: Unknown but bounded errors and system inputs, IEEE Transactions on Automatic Control 13 (1) (1968) 22-28.

[13] L. Uusitalo, A. Lehikoinen, I. Helle, K. Myrberg, An overview of methods to evaluate uncertainty of deterministic models in decision support, Environmental Modelling \& Software 63 (2015) 24-31.

[14] J. Gertler, Fault Detection and Diagnosis, Springer, 2015

[15] V. Puig, J. Quevedo, T. Escobet, A. Stancu, Robust fault detection using linear interval observers, IFAC Proceedings Volumes 36 (5) (2003) 579-584.

[16] R. E. Kalman, R. S. Bucy, New results in linear filtering and prediction theory, Journal of Basic Engineering 83 (1) (1961) 95-108.

[17] C. Combastel, Stable interval observers in C for linear systems with time-varying input bounds, IEEE Transactions on Automatic Control 58 (2) (2013) 481-487.

[18] V. T. H. Le, C. Stoica, T. Alamo, E. F. Camacho, D. Dumur, Zonotopic guaranteed state estimation for uncertain systems, Automatica 49 (11) (2013) 3418-3424.

[19] M. Pourasghar, V. Puig, C. Ocampo-Martinez, Comparison of set-membership and interval observer approaches for state estimation of uncertain systems, in: European Control Conference (ECC), 2016, Denmark, pp. 1111-1116.

[20] B. Noack, F. Pfaff, U. D. Hanebeck, Optimal Kalman gains for combined stochastic and set-membership state estimation, in: The 51st IEEE Conference on Decision and Control (CDC), 2012, pp. 4035-4040.

[21] L. Jaulin, Robust set-membership state estimation; application to underwater robotics, Automatica 45 (1) (2009) $202-206$.

[22] V. Puig, P. Cugueró, J. Quevedo, Worst-case state estimation and simulation of uncertain discrete-time systems using zonotopes, European Control Conference (ECC) (2001) 1691-1697.

[23] J. M. Bravo, T. Alamo, E. F. Camacho, Bounded error identification of systems with time-varying parameters, IEEE Transactions on Automatic Control 51 (7) (2006) 1144-1150.

[24] C. Combastel, A state bounding observer based on zonotopes, in: European Control Conference (ECC), 2003 , pp. $2589-2594$.

[25] S. Ploix, O. Adrot, Parity relations for linear uncertain dynamic systems, Automatica 42 (9) (2006) 1553-1562.

[26] P. M. Frank, X. Ding, Frequency domain approach to optimally robust residual generation and evaluation for model-based fault diagnosis, Automatica 30 (5) (1994) 789-804.

[27] S. Ding, Model-based fault diagnosis techniques: design schemes, algorithms, and tools, Springer Science \& Business Media, 2008.

[28] R. Isermann, Model-based fault-detection and diagnosis-status and applications, Annual Reviews in Control 29 (1) (2005) $71-85$.

[29] A. Ingimundarson, J. M. Bravo, V. Puig, T. Alamo, Robust fault diagnosis using parallelotope-based set-membership consistency tests, in: The 44th IEEE Conference on Decision and Control-European Control Conference (CDC-ECC), 2005, pp. 993-998.

[30] A. Lalami, C. Combastel, Generation of set membership tests for fault diagnosis and evaluation of their worst case sensitivity, IFAC Proceedings Volumes 39 (13) (2006) 569-574.

[31] J. Gertler, Fault detection and isolation using parity relations, Control Engineering Practice 5 (5) (1997) 653-661.

[32] A. R. Kodakkadan, M. Pourasghar, V. Puig, S. Olaru, C. Ocampo-Martinez, V. Reppa, Observer-based sensor fault detectability: About robust positive invariance approach and residual sensitivity, IFAC-PapersOnLine 50 (1) (2017) 5041-5046.

[33] M. Zhong, S. X. Ding, J. Lam, H. Wang, An LMI approach to design robust fault detection filter for uncertain LTI systems, Automatica 39 (3) (2003) 543-550.

[34] M. Sadrnia, J. Chen, R. Patton, Robust fault diagnosis observer design using $h_{\infty}$ optimisation and $\mu$ synthesis, IEE Colloquium on Modelling and Signal Processing for Fault Diagnosis (1996) 1-9.

[35] N. Liu, K. Zhou, Optimal solutions to multi-objective robust fault detection problems, in: 46th IEEE Conference on Decision and Control, 2007, USA, pp. 981-988.

[36] M. Hou, R. J. Patton, An LMI approach to $H_{-} / H_{\infty}$ fault detection observers, in: UKACC International Conference on Control, 1996, UK, pp. 9305-310.

[37] J. Liu, J. L. Wang, G. H. Yang, An LMI approach to minimum sensitivity analysis with application to fault detection, Automatica 41 (11) (2005) 1995-2004.

[38] D. Henry, A norm-based point of view for fault diagnosis. application to aerospace missions, in: 8th European Workshop on Advanced Control and Diagnosis, 2010, Italy, pp. 4-16.

[39] I. M. Jaimoukha, Z. Li, V. Papakos, A matrix factorization solution to the $H_{-} / H_{\infty}$ fault detection problem, Automatica 42 (11) (2006) 1907-1912.

[40] J. L. Wang, G.-H. Yang, J. Liu, An LMI approach to $H_{-}$index and mixed $H_{-} / H_{\infty}$ fault detection observer design, Automatica 43 (9) (2007) $1656-1665$

[41] S. X. Ding, T. Jeinsch, P. M. Frank, E. L. Ding, A unified approach to the optimization of fault detection systems, International journal of adaptive control and signal processing 14 (7) (2000) 725-745.

[42] C. Combastel, Zonotopes and Kalman observers: Gain optimality under distinct uncertainty paradigms and robust convergence, Automatica 55 (2015) 265-273.

[43] C. Combastel, Merging Kalman filtering and zonotopic state bounding for robust fault detection under noisy environment, IFACPapersOnLine 48 (21) (2015) 289-295.

[44] C. Combastel, An Extended Zonotopic and Gaussian Kalman Filter (EZGKF) merging set-membership and stochastic paradigms: Toward non-linear filtering and fault detection, Annual Reviews in Control 42 (2016) 232-243.

[45] J. Meseguer, V. Puig, T. Escobet, J. Saludes, Observer gain effect in linear interval observer-based fault detection, Journal of Process Control $20(8)(2010)$ 944-956.

[46] R. Patton, J. Chen, A review of parity space approaches to fault diagnosis, in: Safeprocess, 1991, pp. 65-81. 
[47] K. H. Johansson, The quadruple-tank process: A multivariable laboratory process with an adjustable zero, IEEE Transactions on Control Systems Technology 8 (3) (2000) 456-465. 